\title{
Global gene expression changes of in vitro stimulated human transformed germinal centre B cells as surrogate for oncogenic pathway activation in individual aggressive $B$ cell lymphomas
}

Alexandra Schrader ${ }^{1,9,12^{*}}$, Katharina Meyer ${ }^{2,7,10}$, Frederike von Bonin ${ }^{1}$, Martina Vockerodt ${ }^{3,11}$, Neele Walther ${ }^{1}$, Elisabeth Hand ${ }^{1,10}$, Antje Ulrich ${ }^{1,8}$, Kamila Matulewicz ${ }^{1,7}$, Dido Lenze ${ }^{4,7}$, Michael Hummel ${ }^{4,7}$, Arnd Kieser ${ }^{5}$, Michael Engelke ${ }^{6}$, Lorenz Trümper ${ }^{1,7,8}$ and Dieter Kube ${ }^{1,7,8,10}$

\begin{abstract}
Background: Aggressive Non-Hodgkin lymphomas (NHL) are a group of lymphomas derived from germinal centre B cells which display a heterogeneous pattern of oncogenic pathway activation. We postulate that specific immune response associated signalling, affecting gene transcription networks, may be associated with the activation of different oncogenic pathways in aggressive Non-Hodgkin lymphomas (NHL).

Methodology: The B cell receptor (BCR), CD40, B-cell activating factor (BAFF)-receptors and Interleukin (IL) 21 receptor and Toll like receptor 4 (TLR4) were stimulated in human transformed germinal centre $B$ cells by treatment with anti IgM F(ab) 2 -fragments, CD4OL, BAFF, IL21 and LPS respectively. The changes in gene expression following the activation of Jak/STAT, NF-KB, MAPK, $\mathrm{Ca}^{2+}$ and PI3K signalling triggered by these stimuli was assessed using microarray analysis. The expression of top 100 genes which had a change in gene expression following stimulation was investigated in gene expression profiles of patients with Aggressive non-Hodgkin Lymphoma (NHL).

Results: algM stimulation led to the largest number of changes in gene expression, affecting overall 6596 genes. While CD40L stimulation changed the expression of 1194 genes and IL21 stimulation affected 902 genes, only 283 and 129 genes were modulated by lipopolysaccharide or BAFF receptor stimulation, respectively. Interestingly, genes associated with a Burkitt-like phenotype, such as MYC, BCL6 or LEF1, were affected by algM. Unique and shared gene expression was delineated. NHL-patients were sorted according to their similarity in the expression of TOP100 affected genes to stimulated transformed germinal centre B cells The algM gene module discriminated individual DLBCL in a similar manner to CD40L or IL21 gene modules. DLBCLs with low module activation often carry chromosomal MYC aberrations. DLBCLs with high module activation show strong expression of genes involved in cell-cell communication, immune responses or negative feedback loops. Using chemical inhibitors for selected kinases we show that mitogen activated protein kinase- and phosphoinositide 3 kinase-signalling are dominantly involved in regulating genes included in the algM gene module.

(Continued on next page)
\end{abstract}

\footnotetext{
* Correspondence: alexandra.schrader@med.uni-goettingen.de

'Department of Haematology and Oncology, University Medical Centre Göttingen, Göttingen, Germany

${ }^{9}$ GRK 1034 of the Deutsche Forschungsgemeinschaft, Göttingen, Germany

Full list of author information is available at the end of the article
} 
(Continued from previous page)

Conclusion: We provide an in vitro model system to investigate pathway activation in lymphomas. We defined the extent to which different immune response associated pathways are responsible for differences in gene expression which distinguish individual DLBCL cases. Our results support the view that tonic or constitutively active MAPK/ERK pathways are an important part of oncogenic signalling in NHL. The experimental model can now be applied to study the therapeutic potential of deregulated oncogenic pathways and to develop individual treatment strategies for lymphoma patients.

Keywords: Gene expression pattern, Lymphoma, Pathway activation

\section{Lay abstract}

Aggressive Non-Hodgkin lymphomas (NHL) are a heterogeneous group of lymphomas derived from germinal centre B cells. $30 \%$ of NHL patients do not respond to treatment. Current criteria to distinguish individual NHL subtypes such as morphology, immunophenotype, and genetic abnormalities do not allow reliable subtype categorization and prediction of treatment response for NHL cases. The pathological mechanisms behind this heterogeneity are poorly understood. Thus there is a need of new and additional methods for stratifying NHL.

The purpose of our studies is to estimate the extent to which distinct signal transduction pathways could be responsible for the differences in gene expression that distinguish individual lymphomas. We postulate that signals associated with the immune response can resemble pathways activated in distinct NHL subtypes.

To gain closer insight into the relevance of distinct cell signaling networks to NHL subtypes, we stimulated human transformed germinal centre B cells with factors known to modify B cell signalling, or which are involved in B cell microenvironment or lymphoma pathogenesis. We discovered that coherent gene expression patterns, related to distinct in vitro stimuli, characterize individual NHLs. Exemplified by an $\alpha I g M$ stimulation we identified signalling pathways dominantly involved in regulating this consistent global gene expression pattern.

We provide an in vitro model system of pathways activated in transformed $B$ cells which allows a better understanding of the global expression changes observed in particular lymphoma subgroups. This model can be used in the future to study the therapeutic potential of oncogenic pathway activation and to develop individual treatment strategies for patients.

\section{Background}

Mature aggressive Non-Hodgkin lymphomas (NHL) are a heterogeneous group of lymphomas most often derived from $B$ cells during the germinal centre B cell reaction [1-3]. Approximately 30 percent of patients with NHL classified as diffuse large B cell lymphoma (DLBCL) do not respond to treatment $[4,5]$. The criteria currently used to distinguish between Burkitt lymphoma
(BL) and DLBCL, is based on differences in morphology, immunophenotype, and genetic abnormalities. These are not reliably reproducible and most importantly the pathological mechanisms behind these criteria are poorly understood [3]. NHL cells proliferate actively and retain many of the immunophenotypic characteristics of germinal centre B lymphocytes. However, they are monoclonal tumour B cells, and display characteristic nonrandom chromosomal abnormalities. Cellular genes thus can be placed under the control of heterologous promoter or enhancer elements and may switch off cellular growth regulation. In contrast, specific combinations of signals for short or long term stimulation are provided to germinal centre $\mathrm{B}$ (GC B) cells through externally derived signals obtained from cells in the microenvironment $[1,6]$.

In peripheral secondary lymphoid organs B cells encounter foreign antigens. Antigen-stimulated B cells can in turn form germinal centres. In the microenvironment of germinal centres B cells need to interact with other cells, such as $\mathrm{T}$ cells, tingible body macrophages, follicular dendritic and reticular cells [1]. Signal transduction pathways initiated through the BCR determine the fate of B cells in dependence of BCR affinity to antigen, concomitant engagement of coreceptors and the differentiation stage of B cells [7]. GC B cells undergo apoptosis if not rescued through GC survival signals. However, unresolved chromosomal translocations and/or permanently deregulated autocrine or paracrine stimulations counteracting these processes can lead to transformation of GC B cells [1]. Within the GC B cell reaction or maintenance of mature $B$ cells additional factors are involved including IL21, CD40L (TNFSF5 / CD154) or tumour necrosis factor superfamily member 13b (BAFF / TNFSF13b / CD257) [2,4-6,8]. In addition, there is evidence for an involvement of pattern recognition receptors in these processes [8]. It is well know from different cell systems that after treating cells with the mentioned stimuli a number of pathways are activated. This includes IL21-mediated modulation of janus-kinase (Jak) and signal transducer and activator of transcription (STAT) or mitogen activated kinases (MAPK)1/2 (Erk1/2) [8]. Furthermore, canonical and non-canonical nuclear factor- $\mathrm{KB}$ (NF-кB), MAPK8/9 (JNK1/2), MAPK14 (p38a) signalling 
is affected through CD40L, non-canonical NF-KB by BAFF, canonical NF- $\mathrm{KB}$ by LPS [8-12]. In addition $\mathrm{Ca}^{2+}$, phosphoinositide 3 kinase (PI3K), Erk1/2, canonical NF-кB, JNK1/2, p38a signalling can be initiated by $B$ cell receptor activation [2,13-16]. In addition, aberrant signalling caused by a defined set of mutations or autocrine and paracrine loops for these pathways have been reported to be important for B cell lymphoma initiation or maintenance $[2,11,17-19]$.

Recent large-scale gene expression profiling of NHL tumour samples revealed a molecular definition for BL, by describing a specific signature. This signature was used to model an index of 'Burkitt-likeness' (mBL-index) and to distinguish BLs from DLBCLs [20,21]. A fundamental question from these studies is the extent to which different pathways could be responsible for the differences in gene expression that distinguish individual DLBCL. We hypothesized that gene transcription networks affected by immune response associated signals resemble oncogenic pathway activity in DLBCL.

So far two major molecular patterns for DLBCLs are described: so called activated $\mathrm{B}$ cell $(\mathrm{ABC})$ like lymphoma and germinal centre B cell (GC B) like lymphoma. They can be complemented by for example host response, stromal or even NF-кB specific gene expression signatures [22-25]. Recent combinations of in vitro cell interventions with systems biology allowed the prediction of potential oncogenic pathways involved in B cell transformation [26-28]. Furthermore, in vitro studies showed that combined STAT3 and NF-кB pathway activities are central to $A B C-$ like lymphoma cells $[22,29,30]$. In addition, there is evidence that aberrant Toll like receptor (TLR) and BCR signalling may be involved affecting $\mathrm{PI} 3 \mathrm{~K}$ and/or MAPK/Erk signalling in addition to NF-KB $[13,18,31,32]$. These data are based mainly on interventions of constitutively activated pathways by knockdown experiments and mutational analysis $[2,13,18]$.

To get more insight into cell signalling networks and their presence in individual human NHL, we utilized human transformed GC B cells. We demonstrate that $B$ cell specific stimuli can be used to identify gene expression changes. This allows a "switch" in gene expression from a steady state level characteristic of BL towards that of DLBCLs. Representative sets of genes (gene modules) are used to describe individual lymphomas. DLBCLs are heterogeneous in the appearance of the magnitude of their gene module activation ranging between "off" and "on". Our data support the view that, for example, tonic and/or activated mitogen activated protein kinase- and phosphoinositide 3 kinase pathway components are part of a signalling network that distinguishes individual DLBCL. Furthermore, a useful in vitro model system to test for individual treatment strategies is offered.

\section{Results and discussion}

Global gene expression changes in human transformed germinal centre B cells stimulated with B cell specific paracrine stimuli

In order to achieve global gene expression changes to describe major pattern of gene expression and to identify pathway activity in aggressive NHL we used as our model system, the BL2 cell line, which is derived from germinal centre B cells [33-35]. BL2 cells were stimulated using CD40L, BAFF, IL21, $\alpha \operatorname{IgM~F}(\mathrm{ab})_{2}$ fragments or lipopolysaccharide (LPS) as described in Material and Methods section (Additional file 1: Supplementary Materials and Methods). These stimuli were chosen, because they are well known mediators of signalling in B cells, involved in GC B cell microenvironment and involved in B cell lymphoma initiation or maintenance [2,11,17-19]. Following stimulation, we wanted to identify gene expression changes which reflect pathways involved in ligand specific signal transduction and pathways potentially active in aggressive NHL. Time points of stimulations were chosen to achieve a signal strong enough to be detected as gene expression change at the whole genome level. Probes of three independent biological experiments were hybridized to U133 plus 2.0 microarrays. Differentially expressed genes were identified using linear models as implemented in the Bioconductor package LIMMA [36]. False discovery rates of differentially expressed genes were calculated according to the Benjamini and Hochberg in a paired-test as described in the Material and Methods section.

Genes with the greatest change in expression and with an adjusted $\mathrm{p}$ value $\leq 0.05$ in response to each stimulus were chosen for further analysis (Table 1 and Additional file 2: Table S1, Additional file 3: Table S2, Additional file 4: Table S3, Additional file 5: Table S4, Additional file 6: Table S5). The top 100 differentially expressed genes are depicted as heatmaps in Figure 1. To our knowledge the only comparable data set available is from human transformed germinal centre B cells (Ramos) which were cultivated on a CD40L expressing

Table 1 Differential expression in human transformed germinal centre B cells in response to B cell-specific stimulations

\begin{tabular}{llllll}
\hline & a-IgM & CD40L & IL21 & LPS & BAFF \\
\hline Upregulated genes & 3039 & 689 & 463 & 114 & 69 \\
\hline Downregulated genes & 3557 & 496 & 439 & 169 & 39 \\
\hline Total number of genes affected & 6596 & 1194 & 902 & 283 & 129 \\
\hline
\end{tabular}

BL2 cells stimulated through algM treatment, by CD40L, IL21, BAFF or LPS. RNA was hybridized onto U133A 2.0 plus Arrays. Differentially expressed genes between stimulated and control cells were identified using linear models as implemented in the bioconductor package LIMMA [36]. False discovery rates for lists of differentially expressed genes were calculated according to Benjamini and Hochberg [38]. Genes were ranked according to their p-value for differential expression from the microarray experiments (adj. $P$ value $\leq 0.05$ ). 


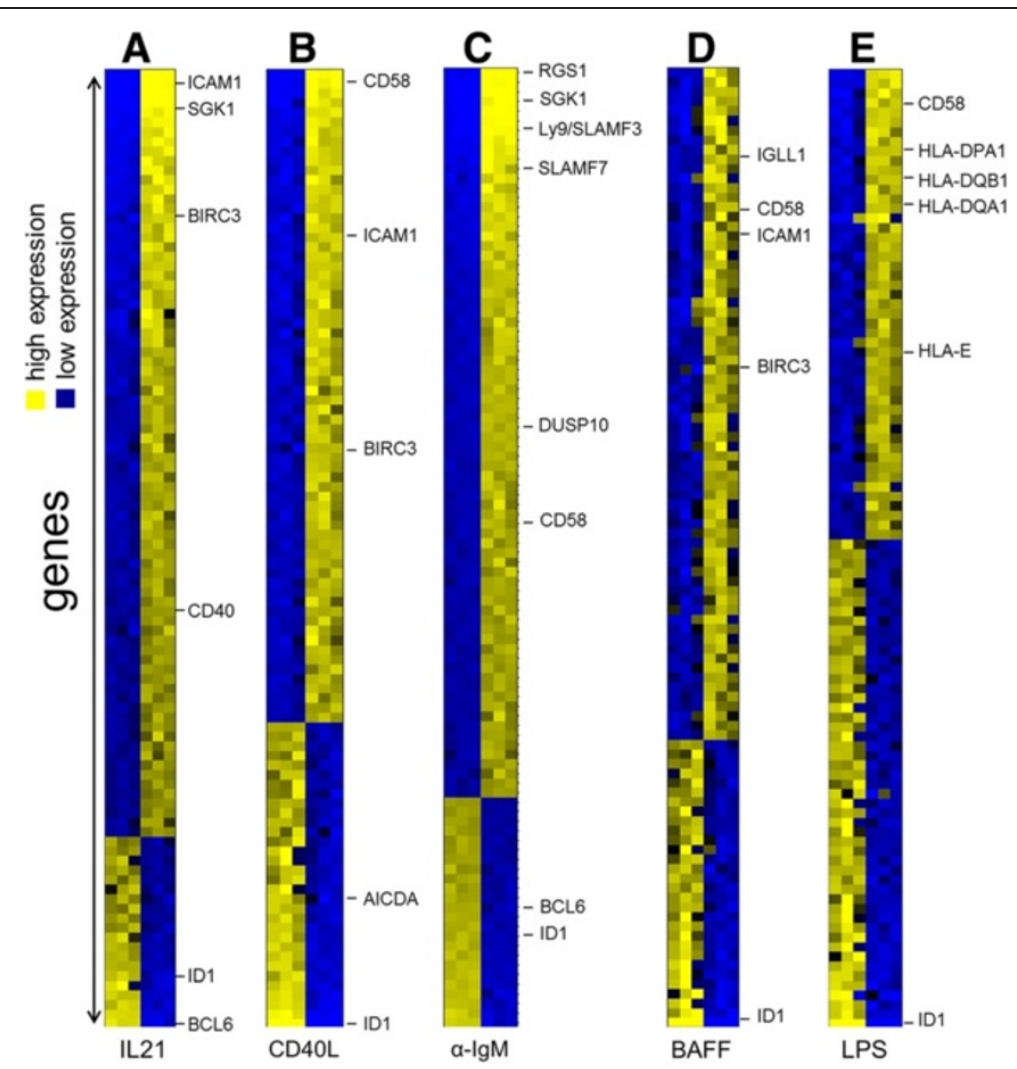

Figure 1 Identification of IL21, CD40L, algM, BAFF and LPS regulated genes in transformed human germinal centre B cells using microarrays. BL2 cell were stimulated with algM F(ab) 2 fragments (3 hrs) (A), IL21 (2 hrs) (B), CD40L (6 hrs) (C), LPS (6 hrs) (D) and BAFF (9 hrs) (E). RNAs from these cells were used for gene expression profiling on Affymetrix HGU-133 plus 2.0 microarray chips. The heatmaps show the most highly changed gene expression (TOP100) (adj. p-value $\leq 0.05$ ) in response to each stimulus. Each row in the heatmaps represents a gene and each column represents a microarray sample. Yellow and blue indicate high and low expression, respectively. Control probes (left column) are shown in comparison to stimulated probes (right column). Additional details are summarized within Table 3 and suppl. Tables 1-5.

feeder cell line for 24 hours [37]. Despite the different experimental conditions, BL2 cells showed similar gene expression changes after exposure to recombinant CD40L for 6 hours (Additional file 7: Figure S1). In contrast, global gene expression changes after B cell receptor activation, for BAFF, LPS or IL21 stimulation have been described using different microarray-platforms. Therefore, a quantitative comparison is difficult. Furthermore, different cell lines or leukocyte cell subsets from a different origin, for example splenic murine B cells or bursal chicken B cells were analysed. A selection of available data is summarized in Additional file 8: Supplemental 1.

\section{Gene set enrichment analyses of global gene expression changes in transformed germinal centre B cells}

Molecular functions, biological processes, cellular components and pathways affected by distinct stimuli were characterized by gene ontology $(\mathrm{GO})$ based gene set enrichment analyses (Additional file 9: Supplemental 2).

$\alpha I g M$ activated genes are linked to MAP kinase activity, phosphatase activity and transmembrane transporter activity. The biological processes affected can be summarized as regulation of immune responses, MAP kinase activity, and programmed cell death, regulation of metabolic processes (glucose / carboxylic acid / organic acid/ amino acid transport) or cell cycle and stress responses.

IL21 activated genes are enriched for gene sets associated with responses to virus and other organisms and cytokine production including type I interferon biosynthetic processes. Furthermore, as for $\alpha \operatorname{IgM}$ activated genes, IL21 affected gene sets are involved in regulation of programmed cell death. The involvement of IL21 activated genes on cytokine signalling could also explain its relation to IкB kinase/NF-кB cascade and NF-кB import into nucleus, gene sets characteristic for Toll like receptor pathways, Jak/STAT and chemokine signalling pathway, but also pathways in cancer are enriched. IL21 suppressed genes are characteristic for nucleotidyltransferase activity, cytoskeletal protein or phospholipid binding thus affecting cell shape, morphogenesis or chemotaxis.

BAFF activated genes are involved in metabolic processes of amino acids and chromatin remodelling, 
whereas downregulated genes are part of lipoprotein metabolic process, protein amino acid acylation.

The CD40L mediated gene expression changes positively affect MHC class I receptor activity and thus antigen processing and presentation of peptide antigen, the regulation of membrane potential, small GTPase mediated signal transduction as well as metabolic processes. In contrast, CD40L suppressed genes are involved in phospholipase activity or negative regulation of transcription.

\section{Gene expression changes in transformed germinal centre $B$ cells of selected microarray results and validation by quantitative real-time PCR}

Stimulation of BL2 cells led to changes in the expression of genes involved in cell-cell communications, including changes in HLA, PECAM, CD1, CD86 or members of the signalling lymphocyte activation molecule family (SLAMF). Interestingly, expression of the $H L A$ group of genes was positively regulated as a result of all stimulations. IL21 affects, for example $H L A-B,-C$ and $-E$ expression. The greatest upregulation was observed for $H L A-D P A 1,-D Q A 1$ and $-D Q B 1$ following BAFF, CD40L and $\alpha I g M$ treatment. Furthermore, CIITA was activated by CD40L and $\alpha I g M$. Expression of the ICAM1 gene, which encodes a protein involved in cellular adhesion and costimulatory signalling and leukocyte trans-endothelial migration, is activated by all the stimuli used (Table 2 and Additional file 2: Table S1, Additional file 3: Table S2, Additional file 4: Table S3, Additional file 5: Table S4, Additional file 6: Table S5). IL21- treatment has the highest impact on ICAM1 activation [39]. CD58, a ligand of CD2, is activated by CD40L and $\alpha \operatorname{IgM}$ treatment [40].

SLAMF-associated proteins are important immunomodulatory receptors with roles in cytotoxicity, humoral immunity, autoimmunity, cell survival, lymphocyte development, and cell adhesion [41]. Whereas SLAMF1, 3 and 7 are strongly upregulated by BCR crosslinking, SLAMF6 is inhibited. This inhibition is most prominent in response to $\alpha \operatorname{IgM}$. In contrast, CD40L treatment is associated with a decreased SLAMF3 expression.

Defined elements of the chemokine system are specifically affected: IL21 upregulates CCR7, CXCR5 and CXCL10, CD40L modulates the expression of CCL5 (increased), CCL17 (increased), CXCR7 (decreased) and CXCL10 (increased), whereas $\alpha \mathrm{IgM}$ treatment affects CCR7 (increased), CXCR7 (decreased) and CXCL1O (increased). The chemokine receptor CCR7, involved in germinal centre $\mathrm{B}$ cell homing is affected by CD40L but much stronger through $\alpha$ IgM [42]. CCR7 plays a pivotal role in homing of tumour cells into lymphomasupporting niches in secondary lymphoid organs [43]. The chemokine CXCL1O is involved in chemotaxis for monocytes and $\mathrm{T}$ lymphocytes and has been reported to play an important role in the pathogenesis of tissue necrosis and vascular damage [44].

The expression of the inhibitor of DNA binding 1 (ID1) is inhibited in response to IL21, CD40L, $\alpha I g M$, BAFF or LPS treatment. The Id proteins are inhibitors of the basic-helix-loop-helix (bHLH) transcription factors [45]. In the $\mathrm{B}$ cell lineage, the ID1 gene is usually expressed in pro$B$ cells and down regulated during differentiation [46]. Interestingly, inhibitors of DNA binding 1, 3 or 4 are inhibited by several stimulations. ID3 expression is activated by $\alpha I g M$, whereas the other stimuli are leading to an inhibition of ID3. ID4 expression is not affected by IL21, whereas in all other cases it is inhibited.

The expression of BCL6, which is a central GC B cell reaction regulator, is inhibited in response to all stimuli [47]. However, the greatest effect was seen following treatment of cells with IL21 and $\alpha$ IgM. Furthermore, BCL6 interacting proteins, BCOR or BCL11A are also affected, by $\alpha \operatorname{IgM}$ or CD40L treatment. Interestingly, this $B C L 6$ downregulation is accompanied by increased expression of CXCL10 comparable to that described by Shaffer and colleagues [48]. In addition, IRF4 is upregulated in response to all stimuli although for BAFF this was not significant. Termination of the GC reaction requires IRF4 as well as the transcriptional repressor Blimp1. IRF4 acts as a crucial transcriptional 'switch' in the generation of functionally competent plasma cells [49]. However, BLIMP1 (PRDM1) is only affected by IL21 (Additional file 3: Table S2) [50]. In addition, LMO2 (LIM domain only 2) is activated by $\alpha \operatorname{IgM}$ and IL21, a factor which also plays a central and crucial role in hematopoietic development and is highly conserved [51]. HGAL (GCET2) acting in concert with for example LMO2 or Bcl6 is suppressed by $\alpha \mathrm{IgM}$ and CD40L treatment [52]. Interestingly, the expression of both AICD and RAG2 is inhibited by $\alpha \operatorname{IgM}$ treatment.

Regarding the GO-analysis, genes involved in programmed cell death mainly affected by CD40L, $\alpha \operatorname{IgM}$ and to some extend also by IL21. Thus, we observed changes in gene expression for example for $B C L 2$, BCL2A1 (BFL), BCL2L1 (BclXl), BCL2L11 (BIM), BCL2L12, CFLAR, FAS or MCL1).

Gene expression changes in response to IL21, CD40L, $\alpha \operatorname{IgM}, \mathrm{BAFF}$ and LPS were also measured by quantitative real time PCR (Figure 2). As exemplified for ICAM1, CD58, CCR7, CXCL10, ID1, BCL6, MYC, RGS1, DUSPs and SLAMF members (Figure 2A-O) an overall good agreement of qRT-PCR data with the microarray data is observed.

\section{Elements of the Wnt pathway are affected by in vitro interventions}

LEF1 was recently defined as a signature gene in defining the index of Burkitt-likeness [20]. Thus, we investigated 
Table 2 Gene expression changes in human transformed germinal centre B cells of selected microarray results

\begin{tabular}{|c|c|c|c|c|c|c|}
\hline Gene symbol & $\log _{2} F C$ & & & & & Suggested function or pathway \\
\hline & a-lgM & CD40L & IL21 & BAFF & LPS & \\
\hline CIITA & 0,79 & 0,81 & - & - & - & Antigen presentation \\
\hline$H L A-B$ & 0,32 & 0,83 & 0,26 & 0,57 & 0,51 & Antigen presentation \\
\hline$H L A-C$ & 0,34 & 0,71 & 0,28 & 0,52 & 0,5 & Antigen presentation \\
\hline HLA-DPA1 & 0,81 & 0,62 & - & - & 0,84 & Antigen presentation \\
\hline HLA-DQA1 & 1,54 & 1,13 & - & 0,75 & 0,74 & Antigen presentation \\
\hline$H L A-D Q B 1$ & 2,2 & 0,99 & - & 0,77 & 0,72 & Antigen presentation \\
\hline HLA-E & - & - & 0,64 & - & 0,54 & Antigen presentation \\
\hline$B C L 11 A$ & $-0,82$ & - & $-0,34$ & - & - & apoptosis \\
\hline$B C L 2$ & 1,63 & - & - & - & - & apoptosis \\
\hline$B C L 2 A 1$ & 4,3 & - & 1,93 & - & - & apoptosis \\
\hline$B C L 2 L 1$ & 0,41 & - & 0,89 & - & - & apoptosis \\
\hline$B C L 2 L 11$ & 0,43 & - & 0,44 & - & - & apoptosis \\
\hline$B C L 2 L 12$, & 0,34 & - & - & - & - & apoptosis \\
\hline CFLAR & $-0,43$ & 0,74 & 0,54 & 0,45 & - & apoptosis \\
\hline FAS & 0,91 & 0,83 & 0,89 & - & - & apoptosis \\
\hline MCL1 & 0,57 & - & 1,01 & - & - & apoptosis \\
\hline$C D 1 C$ & 2,67 & - & - & - & - & Cell-cell communication, immune response \\
\hline CD58 & 1,94 & 1,52 & - & - & 0,84 & Cell-cell communication, immune response \\
\hline CD86 & 1,16 & $-0,54$ & - & - & $-0,34$ & Cell-cell communication, immune response \\
\hline ICAM1 & 0,75 & 1,01 & 2,91 & 0,65 & 0,48 & Cell adhesion \\
\hline PECAM & $-0,31$ & $-0,56$ & - & - & $-4,9$ & Cell adhesion \\
\hline SLAMF1 & 0,47 & - & - & - & - & Cell-cell communication, immune response \\
\hline SLAMF3 & 4,14 & $-0,36$ & - & - & $-0,42$ & Cell-cell communication, immune response \\
\hline SLAMF6 & $-1,1$ & $-0,48$ & $-0,65$ & - & - & Cell-cell communication, immune response \\
\hline SLAMF7 & 2,07 & - & - & - & - & Cell-cell communication, immune response \\
\hline DUSP10 & 2,17 & - & 0,89 & - & - & Phosphatase activity, Negative regulation of MAPK \\
\hline DUSP2 & 2,99 & - & 1,09 & - & - & Phosphatase activity, Negative regulation of MAPK \\
\hline DUSP22 & 0,74 & 0,98 & - & - & 0,53 & Phosphatase activity, Negative regulation of MAPK \\
\hline RGS1 & 5,5 & - & - & - & - & Negative regulation of G-protein couppled receptors \\
\hline CXCL10 & - & 0,4 & 1,63 & - & - & chemokine \\
\hline CCR7 & 3,6 & - & 0,57 & - & - & Chemokine receptor \\
\hline CXCR5 & 0,38 & - & 1,14 & - & - & Chemokine receptor \\
\hline$B C L 6$ & $-2,03$ & $-0,48$ & $-1,94$ & - & - & Gene regulation, B cell differentiation \\
\hline$B C O R$ & $-0,69$ & - & - & - & - & Gene regulation \\
\hline GCET2 & $-0,95$ & $-0,45$ & - & - & - & Gene regulation, B cell differentiation \\
\hline ID1 & $-2,12$ & $-2,04$ & $-1,53$ & $-2,1$ & $-1,9$ & Gene regulation \\
\hline ID2 & 0,74 & - & - & - & - & Gene regulation \\
\hline ID3 & 0,72 & -1 & $-1,58$ & $-0,56$ & $-0,82$ & Gene regulation \\
\hline ID4 & $-2,71$ & $-0,83$ & $-0,78$ & 0,91 & $-0,79$ & Gene regulation \\
\hline IRF4 & 1,53 & 0,46 & 2,14 & - & 0,58 & Gene regulation, B cell differentiation \\
\hline LMO2 & 1,45 & - & 0,53 & - & - & Gene regulation, B cell differentiation \\
\hline PRDM1 & - & - & 0,47 & - & - & Gene regulation, B cell differentiation \\
\hline$A / C D$ & $-0,76$ & $-0,34$ & 0,82 & . & . & Somatic hypermutation and class switch recombination \\
\hline AXIN1 & 0,4 & - & - & - & - & Wnt pathway \\
\hline$B C L 9$ & $-0,44$ & - & - & - & - & Wnt pathway \\
\hline$\overline{D V L 1}$ & $-0,3$ & - & - & - & - & Wnt pathway \\
\hline
\end{tabular}


Table 2 Gene expression changes in human transformed germinal centre B cells of selected microarray results (Continued)

\begin{tabular}{|c|c|c|c|c|c|c|}
\hline FLI1 & $-0,6$ & - & - & - & - & Wnt pathway \\
\hline FRAT1 & $-0,37$ & - & - & - & - & Wnt pathway \\
\hline FRAT2 & $-0,96$ & - & - & - & - & Wnt pathway \\
\hline FRZB & $-0,72$ & $-0,39$ & - & - & - & Wnt pathway \\
\hline$\overline{F Z D 2}$ & $-0,56$ & $-0,29$ & - & - & $-0,28$ & Wnt pathway \\
\hline FZD3 & $-0,81$ & - & - & - & - & Wnt pathway \\
\hline FZD6 & 0,32 & - & $-0,3$ & - & - & Wnt pathway \\
\hline Kremen2 & - & 0,35 & - & - & - & Wnt pathway \\
\hline LEF1 & $-0,85$ & - & - & - & $-0,37$ & Gene regulation, Wnt pathway \\
\hline Myc & $-0,72$ & - & - & - & - & Gene regulation, Wnt pathway \\
\hline PYGO1 & 1,13 & - & - & - & - & Wnt pathway \\
\hline RAG2 & $-0,55$ & & & & & VDJ-recombination \\
\hline TCF7 & - & 0,44 & - & - & - & Wnt pathway \\
\hline$\overline{T L E 3}$ & $-0,66$ & $-0,48$ & - & - & $-0,35$ & Wnt pathway \\
\hline WNT10 & $-1,37$ & - & - & - & - & Wnt pathway \\
\hline WNT3 & 0,4 & - & - & - & - & Wnt pathway \\
\hline WNT5a & $-0,72$ & 0,93 & - & - & - & Wnt pathway \\
\hline WNT5B & - & 0,5 & - & - & - & Wnt pathway \\
\hline
\end{tabular}

A specific regulation of genes encoding for proteins involved in cellular adhesion and costimulatory signalling, cell-cell communications or antigen presentation and immunomodulation, cytotoxicity, humoral immunity, autoimmunity, cell survival, lymphocyte development, and cell adhesion, chemokine pathways, apoptosis or Wnt-signaling and GC B cell reaction regulation is revealed. Data are presented as $\log _{2} \mathrm{FC}$ from one probe set. Additional details are found in Additional file 2: Table S1, Additional file 3: Table S2, Additional file 4: Table S3, Additional file 5: Table S4, Additional file 6: Table S5.

changes in the expression of Wnt-pathway components. Interestingly, $\alpha I g M$ stimulation led to reduced LEF1 expression (Figure 2P). The same was observed for BCL9. PYGO1 expression was elevated in response to BCR activation. This was verified by qRT-PCR analysis. Comparable to the stimulation effect on $L E F 1$ expression, we verified the dominant effect of $\alpha \operatorname{IgM}$ treatment on $B C L 9$ (down) and PYGO1 (up) (Figure 2Q, R). Furthermore, AXIN1, FZD2, 3, 6, FRAT1, 2 or DVL1, FLI1, TLE3, FRZB, WNT3, $5 A, 10$ were changed to a lesser extent by $\alpha \operatorname{IgM}$. This is an important observation because Wnt5a produced by follicular dendritic cells affects the B cell differentiation program of germinal centre B cells [53]. The expression of FZD6 and WNT5a are modulated by IL21 and TLE3 by LPS. In addition, CD40L modulates the expression of FRZB, KREMEN2, TCF7, TLE3 and WNT5A (Table 2). Therefore, we conclude that $\alpha \operatorname{IgM}$ stimulation affects major signature genes such as MYC and LEF1 defining the index of Burkitt-likeness [20].

\section{IL21, CD40L, algM, BAFF and LPS affected gene} expression changes: similarity and uniqueness

In order to describe similarities in gene expression the global responses to the stimuli were analysed by the Ordered List approach (Figure 3) [54]. In this approach, genes were ranked according to their fold change in response to respective stimulation. Pairwise comparisons of top and bottom ranks of lists representing IL21,
CD40L, $\alpha$ IgM, BAFF and LPS responses were plotted. We observed a high overlap of genes responding in the same manner for each pairwise comparison $(\mathrm{p}<0.005)$. This can be seen in Figure 3 by the difference between the blue line, representing the number of overlapping genes at the corresponding position of the gene lists given and the orange area giving the expected size of a random overlap. The gene lists are also compared in reversed order represented by the green line. The genes are summarized within the supplementary information (Additional file 10: Table S6, Additional file 11: Table S7, Additional file 12: Table S8, Additional file 13: Table S9, Additional file 14: Table S10, Additional file 15: Table S11, Additional file 16: Table S12, Additional file 17: Table S13, Additional file 18: Table S14, Additional file 19: Table S15). The strongest overlap was observed for IL21 and $\alpha \operatorname{IgM}$. This is somehow surprising since it was suggested that the shared $\mathrm{NF \kappa B}$ driven gene expression changes mediated by LPS, CD40L, $\alpha$ IgM or BAFF would be dominant in defining the major pattern of gene expression changes. However, the strong overlap of IL21 with $\alpha \operatorname{IgM}$ is also reflected in the GO analysis, showing that IL21 and $\alpha$ IgM gene expression changes are enriched for positive regulation of the ІкВ kinase/NF-кB cascade, RNA metabolic processes or immune system processes but also DNA-repair (Additional file 20: Supplemental 3). The shared functions of CD40L and $\alpha I g M$ affected genes are for example characterized by immune response, antigen 


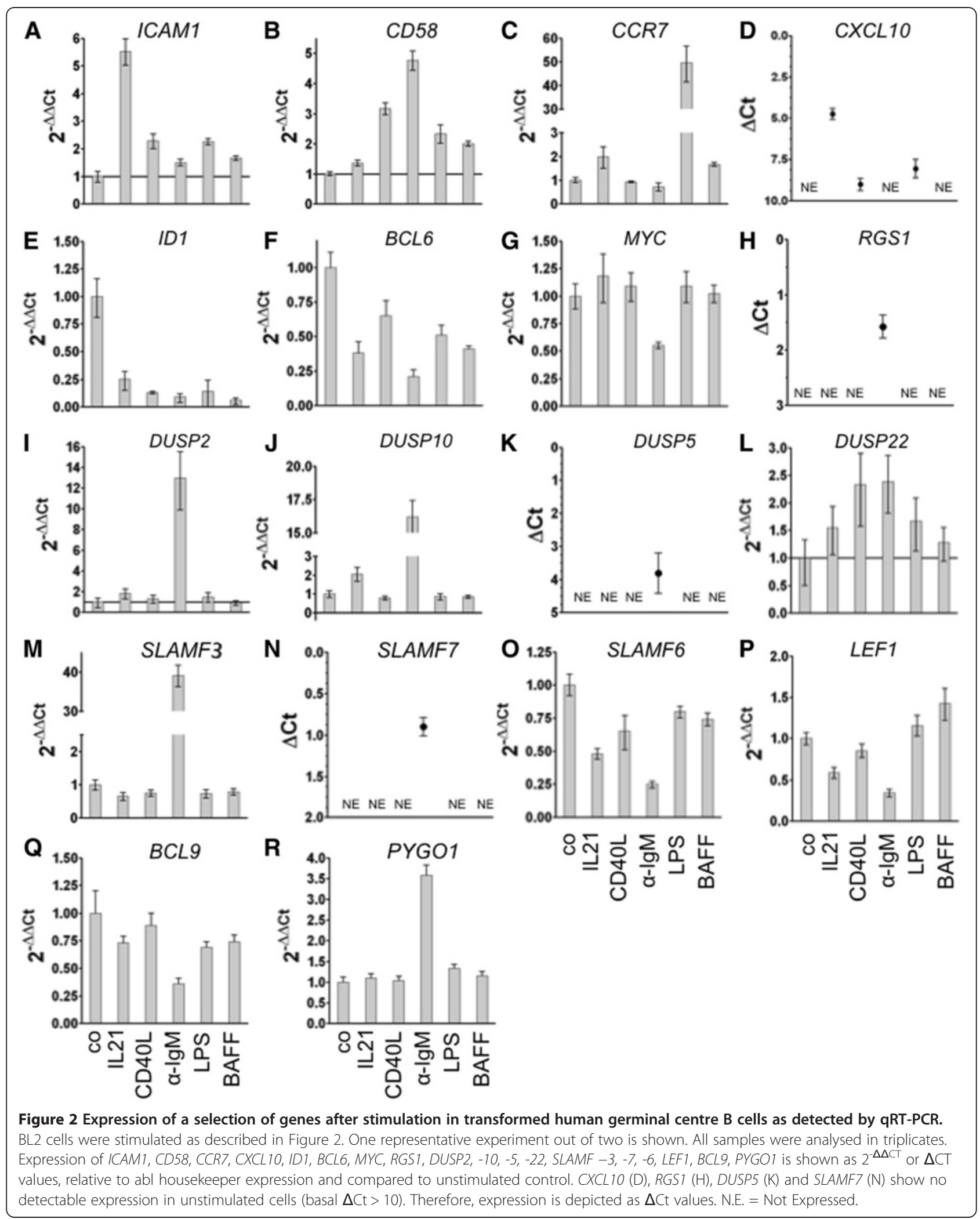



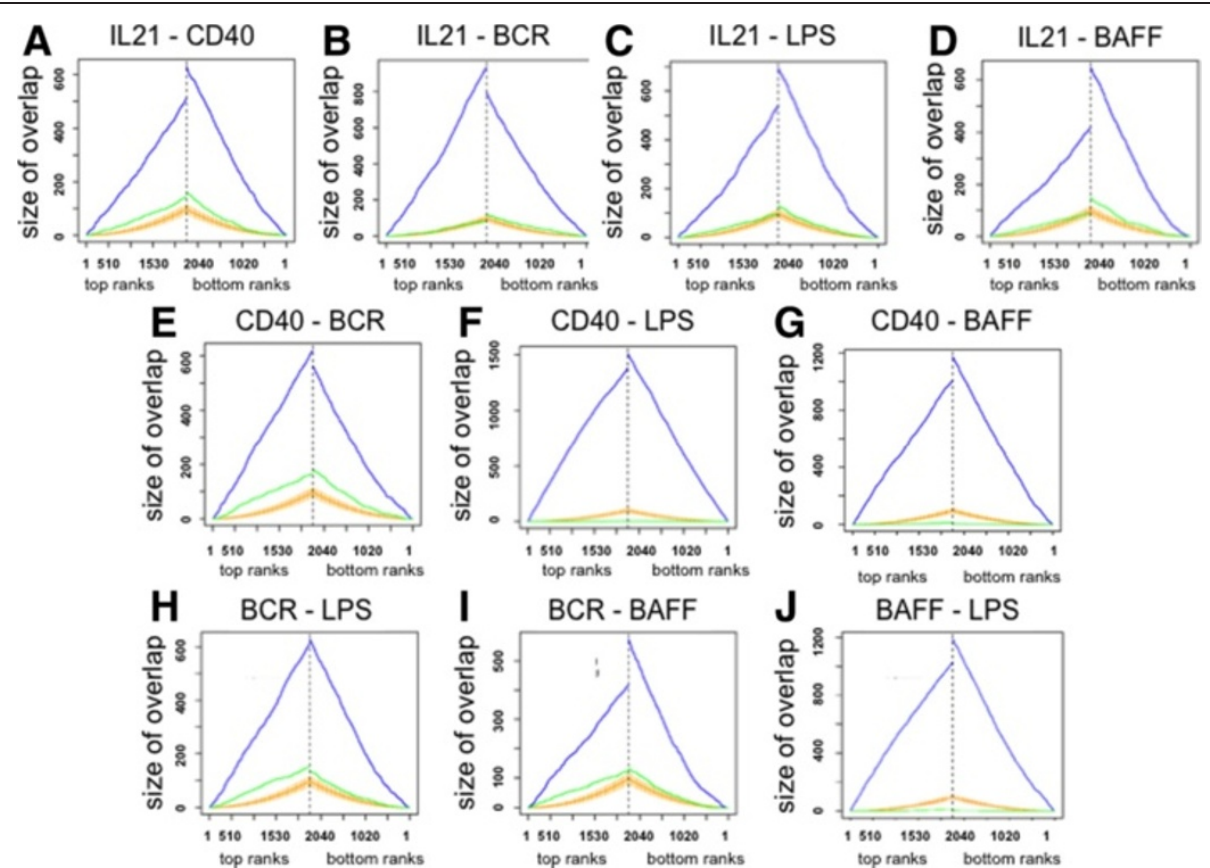

Figure 3 Overlap in global gene expression of IL-21, CD40L, algM, BAFF and LPS affected genes. BL2 cells were stimulated and data were processed as described in the text. The overlaps of differentially expressed gene lists at both ends were compared using the "Ordered List package" [55]. Genes affected by the stimuli assigned on top of each plot are compared. The gene lists are compared in the same (blue line) or reversed order (green line). The $y$-axis gives the size of the overlap in the number of genes of the top n-genes ( $x$-axis) in both gene lists. The orange line gives the expected size of a random overlap, and the vertical orange lines indicate $95 \%$ probability intervals of random overlaps. All overlaps show a high statistical significance $(a<0.005)$. Additional details are summarized within Additional file 10: Table S6, Additional file 11: Table S7, Additional file 12: Table S8, Additional file 13: Table S9, Additional file 14: Table S10, Additional file 15: Table S11, Additional file 16: Table S12, Additional file 17: Table S13, Additional file 18: Table S14, Additional file 19: Table S15.

processing and presentation or positive regulation of B cell activation, BMP signalling pathway and phosphate metabolic processes.

In addition, we describe genes that are specifically affected only by one of the utilized stimuli (uniqueness) (Additional file 21: Table S16). Interestingly, those genes which are dominantly affected by $\alpha \operatorname{IgM}$ treatment are part of biological processes such as nucleic acid binding, PI3K regulator activity, regulation of cell cycle or metabolic processes, Wnt receptor signalling pathways and response to hypoxia (Additional file 22: Supplemental 4).

Therefore, our data now provide a comprehensive collection of gene expression changes induced by different physiological stimuli. These data sets can be used for a better understanding of gene expression changes in $\mathrm{B}$ cell signalling and lymphoma as we will show below. An in vitro model system will be tested to investigate pathway activations in individual DLBCL.

\section{Coherent gene expression of algM affected genes characterizes individual NHL}

To further underpin the functional relevance of the gene expression changes observed following treatment with the stimuli, we investigated whether the change in expression of these genes is comparable to primary NHL. Two independent patient cohorts were included. The gene expression profile from 219 primary tumour samples described by Hummel et al. (MMML1 cohort) and 99 published by Dave et al. (LLMPP cohort) were compared to the gene expression changes described above [20,21]. The genes were summarized in Table 3. In some cases less genes were used because they were missing on the microarrays used for lymphoma gene expression analysis.

$\alpha \operatorname{IgM}$ driven gene expression changes had the greatest absolute fold changes therefore we started with these. The expression levels of a list of 100 genes with a FDR $<0.1$ were examined in clinical lymphoma samples. Their joint expression was estimated using a standard additive model fitted by Tuckey's median polish procedure. These gene groups are further referred to as gene modules. The $\alpha \operatorname{IgM}$ gene module can be used to differentiate BLs from DLBCLs shown in a heatmap (Figure 4A) [20]. On top of the heatmap are labels for the molecular classification (index of Burkitt-likeness, molecular ABC/GCB-classification) and the presence of a chromosomal translocation of $M Y C$. Patients from the MMML1 cohort are sorted according to their increase in the expression of genes from the gene module. On the right part of the heatmap 
Table 3 Most prominent altered genes after in vitro interventions of human transformed germinal centre B cells investigated in gene expression profiles of primary samples of aggressive Non-Hodgkin Lymphoma

\begin{tabular}{|c|c|c|c|c|c|c|c|c|c|c|}
\hline & BAFF & & CD40L & & anti-lgM & & IL21 & & LPS & \\
\hline & Gene symbols & $\log 2 \mathrm{FC}$ & Gene symbols & $\log 2 \mathrm{FC}$ & Gene symbols & $\log 2 \mathrm{FC}$ & Gene symbols & $\log 2 \mathrm{FC}$ & Gene symbols & $\log 2 \mathrm{FC}$ \\
\hline 1 & ID1 & $-2,07$ & ID1 & $-2,007$ & CYP26A1 & $-2,752$ & BCL6 & $-1,904$ & ID1 & $-1,9$ \\
\hline 2 & ID4 & $-0,92$ & C3orf37 & $-1,432$ & ID4 & $-2,687$ & $\mathrm{SOX}^{*}$ & $-1,805$ & RGS18* & $-0,853$ \\
\hline 3 & BNIP3 & $-0,79$ & SMAD 1* & $-1,182$ & BEST3* & $-2,41$ & DNAJB4 & $-1,589$ & C3orf37 & $-0,85$ \\
\hline 4 & TXNIP & $-0,74$ & DEPTOR & $-1,159$ & IL7R* & $-2,399$ & ID3 & $-1,534$ & DDIT4 & $-0,83$ \\
\hline 5 & BNIP3L & $-0,72$ & CAB39L & $-1,086$ & BMP7 & $-2,373$ & DDIT4 & $-1,514$ & ID3 & $-0,811$ \\
\hline 6 & PFKFB4* & $-0,71$ & CD83 & $-1,017$ & $50 \times 4$ & $-2,343$ & ID1 & $-1,493$ & METTL7A & $-0,8$ \\
\hline 7 & GPER & $-0,68$ & METTL7A & $-1,005$ & TNFSF8* & $-2,264$ & RGCC & $-1,239$ & CAB39L* & $-0,799$ \\
\hline 8 & PNOC & $-0,67$ & ANKRD36BP2* & $-0,983$ & RNF144B* & $-2,213$ & IL7R & $-1,152$ & TXNIP & $-0,786$ \\
\hline 9 & RGS18* & $-0,66$ & ID3 & $-0,983$ & DNAJB4 & $-2,156$ & HEY2* & $-1,136$ & BNIP3 & $-0,776$ \\
\hline 10 & P4HA1 & $-0,654$ & ENPP3* & $-0,937$ & ID1 & $-2,093$ & VEGFA & $-1,115$ & ID4 & $-0,776$ \\
\hline 11 & CCNG2 & $-0,635$ & IRF8 & $-0,924$ & BCL6 & $-2,037$ & GADD45A & $-0,982$ & CCNG2 & $-0,721$ \\
\hline 12 & HILPDA & $-0,631$ & RGS18* & $-0,919$ & $\mathrm{RHOH}$ & $-2,005$ & CYTIP & $-0,93$ & PFKFB4* & $-0,709$ \\
\hline 13 & ID2 & $-0,585$ & FAM167A* & $-0,909$ & RGCC & $-1,946$ & PCDH9 & $-0,914$ & KDM3A & $-0,69$ \\
\hline 14 & SAMD13* & $-0,582$ & AICDA & $-0,864$ & NCKAP1 & $-1,857$ & CCNG2 & $-0,889$ & HILPDA* & $-0,678$ \\
\hline 15 & CD24 & $-0,578$ & GPER & $-0,852$ & IKZF1* & $-1,845$ & TXNIP & $-0,872$ & FAM167A* & $-0,673$ \\
\hline 16 & ID3 & $-0,564$ & ID4 & $-0,835$ & $\mathrm{HEY} 2^{*}$ & $-1,761$ & $\mathrm{BICD} 2$ & $-0,868$ & PTPRC & $-0,665$ \\
\hline 17 & C3orf37 & $-0,56$ & PDZRN4 & $-0,809$ & PCDH9 & $-1,751$ & RGS18* & $-0,861$ & STAT2* & $-0,649$ \\
\hline 18 & GPR18 & $-0,541$ & BNIP3 & $-0,785$ & $50 \times 2^{*}$ & $-1,723$ & ANKRD37* & $-0,852$ & SAMD13* & $-0,631$ \\
\hline 19 & ALDOC & $-0,516$ & VEGFA & $-0,777$ & MYCT1* & $-1,716$ & TRIM8 & $-0,836$ & ENPP3* & $-0,609$ \\
\hline 20 & BBOX1 & $-0,516$ & TP53INP1* & $-0,776$ & RGS18* & $-1,715$ & KIAA0907 & $-0,786$ & SMAD1* & $-0,593$ \\
\hline 21 & CD83 & $-0,509$ & PTPRC & $-0,77$ & SMAD1 & $-1,679$ & NEDD9 & 0,787 & P4HA1 & $-0,593$ \\
\hline 22 & POLD4 & $-0,494$ & RASSF6* & $-0,767$ & MLLT3 & $-1,656$ & RELB & 0,79 & VEGFA & $-0,592$ \\
\hline 23 & HBP1 & $-0,484$ & CCNG2 & $-0,761$ & KIF20A & $-1,631$ & PAPD5* & 0,791 & $\mathrm{KLRC} 3$ & $-0,591$ \\
\hline 24 & FAM167A* & $-0,476$ & CLEC2B & $-0,74$ & FAM214A* & $-1,612$ & $Z N F X 1^{*}$ & 0,794 & GPER & $-0,588$ \\
\hline 25 & $\mathrm{EV} I 2 \mathrm{~A}$ & $-0,476$ & BMP7 & $-0,735$ & $\mathrm{BCL} 2$ & 1,618 & B3GNT2* & 0,797 & ZNF385B* & $-0,588$ \\
\hline 26 & TP53INP1* & $-0,474$ & TLR1 & $-0,732$ & PSAT1* & 1,62 & HIF1A & 0,814 & TP53INP1* & $-0,583$ \\
\hline 27 & YPEL5 & $-0,467$ & SATB1 & $-0,731$ & LOC285628* & 1,637 & KIF26B & 0,814 & UBE2H* & $-0,581$ \\
\hline 28 & TRIM22 & $-0,464$ & BTG2 & $-0,726$ & FAIM3 & 1,641 & MMD & 0,82 & TRIM22 & $-0,571$ \\
\hline 29 & TLR1 & $-0,463$ & $\mathrm{PCDH} 9$ & $-0,725$ & MAP2K3 & 1,644 & $\mathrm{AlCDA}^{*}$ & 0,822 & PIK3C2B & $-0,571$ \\
\hline 30 & CLEC2B & $-0,463$ & PRKACB & $-0,724$ & PPP1R15A & 1,662 & EPSTI1* & 0,837 & STAP1 & $-0,564$ \\
\hline 31 & $\mathrm{HLA}-\mathrm{C}$ & 0,457 & GPM6A & $-0,721$ & FAM208B & 1,674 & RAB11FIP1 & 0,844 & BNIP3L & $-0,558$ \\
\hline 32 & ACTR3* & 0,46 & STAT2* & $-0,72$ & $\mathrm{SIAH2}$ & 1,676 & RAB30 & 0,845 & MYBL1 & $-0,556$ \\
\hline 33 & $\mathrm{NAF}^{*}$ & 0,46 & CLIP2 & 0,721 & HIVEP3* & 1,679 & SIN3A* & 0,847 & GPM6A & $-0,549$ \\
\hline 34 & PSMD12 & 0,461 & ARHGAP17 & 0,727 & METRNL* & 1,711 & PLEK & 0,854 & ANKRD37* & $-0,548$ \\
\hline 35 & NUDT5* & 0,461 & CFLAR & 0,74 & HERPUD1 & 1,727 & IFITM1 & 0,863 & SPG11 & $-0,537$ \\
\hline 36 & TESC & 0,463 & FYTTD1* & 0,743 & KLF10 & 1,749 & PRDM2 & 0,885 & NARF & $-0,536$ \\
\hline 37 & CCAR1* & 0,464 & HLA-DMB & 0,747 & LTA & 1,753 & DUSP10 & 0,888 & $A B C A 1$ & $-0,531$ \\
\hline 38 & ANKRD33B* & 0,465 & MTMR10* & 0,749 & GPR18 & 1,769 & FAM100B* & 0,892 & SEC14L1 & $-0,531$ \\
\hline 39 & RILPL2* $^{*}$ & 0,466 & HLA-F & 0,754 & IFRD1 & 1,777 & FAS & 0,894 & IL7R & $-0,522$ \\
\hline 40 & PLCB1 & 0,467 & PLEK & 0,757 & CD274* & 1,785 & IL7 & 0,896 & $\mathrm{H} 1 \mathrm{FX}$ & $-0,52$ \\
\hline 41 & NFKBIE & 0,47 & PPP1R9B* & 0,759 & SLC3A2 & 1,79 & CD69 & 0,921 & ASF1A & $-0,511$ \\
\hline 42 & DDX21 & 0,47 & TESC & 0,764 & HSPA5 & 1,8 & B4GALT1* & 0,923 & GLCCI1* & $-0,509$ \\
\hline 43 & TAPBP & 0,471 & IGLL1 & 0,765 & ATF3 & 1,834 & BCL2L1 & 0,924 & $\mathrm{EVI} 2 \mathrm{~A}$ & $-0,508$ \\
\hline 44 & RBBP6 & 0,474 & SNX20* & 0,765 & DENND4A & 1,848 & CD40 & 0,934 & VGLL4 & $-0,507$ \\
\hline 45 & SOGA2 & 0,474 & CREM $^{*}$ & 0,77 & ARHGAP25 & 1,849 & TET3* & 0,951 & PRKACB & $-0,503$ \\
\hline
\end{tabular}


Table 3 Most prominent altered genes after in vitro interventions of human transformed germinal centre B cells investigated in gene expression profiles of primary samples of aggressive Non-Hodgkin Lymphoma (Continued)

\begin{tabular}{|c|c|c|c|c|c|c|c|c|c|c|}
\hline 46 & HINT1* & 0,474 & IKBKE & 0,782 & TOR3A & 1,85 & SOCS1 & 0,962 & LRP4 & $-0,499$ \\
\hline 47 & SRRM1 & 0,475 & FAS & 0,788 & IRF2BP2* & 1,868 & NFKBID* & 0,983 & PLCXD1 & $-0,498$ \\
\hline 48 & PHACTR4 & 0,475 & CIITA & 0,788 & SLC30A1 & 1,886 & SRSF5 & 0,996 & CLEC2B & $-0,497$ \\
\hline 49 & AK3* & 0,476 & TAP1 & 0,789 & TNF & 1,905 & MASTL* & 1,011 & LOC440864* & $-0,494$ \\
\hline 50 & KPNB1 & 0,477 & NOTCH2NL* & 0,797 & $\mathrm{CTH}$ & 1,91 & TLR7 & 1,036 & SLC44A1* & $-0,493$ \\
\hline 51 & GTF2H2B* & 0,477 & RFX5 & 0,806 & APOBEC3B & 1,928 & MCL1 & 1,04 & TSPAN11* & $-0,484$ \\
\hline 52 & TAF1D & 0,477 & FDXR & 0,81 & NFKB1 & 1,94 & PIM2 & 1,06 & BTN2A2 & 0,488 \\
\hline 53 & CSF2RB & 0,479 & NFKBIE & 0,827 & CD58 & 1,946 & IER2 & 1,067 & NFKBIE & 0,493 \\
\hline 54 & MYBBP1A & 0,481 & MIR155HG* & 0,829 & NFKBIE & 1,958 & ZFP36L1 & 1,072 & HLA-C & 0,495 \\
\hline 55 & $Y Y 1^{*}$ & 0,483 & LOXL2 & 0,829 & NAB2 & 1,984 & IFIT2* & 1,072 & LSS & 0,502 \\
\hline 56 & FLVCR1* & 0,488 & HLA-B & 0,831 & JUND & 2,024 & SNX11 & 1,078 & FDXR & 0,502 \\
\hline 57 & NAA16 & 0,49 & VAV2* & 0,832 & ARL4C & 2,026 & TAP1 & 1,086 & $\mathrm{KLHDC5}{ }^{*}$ & 0,505 \\
\hline 58 & SRRT & 0,491 & ZNF385C* & 0,836 & UPP1 & 2,029 & JUNB & 1,088 & HLA-B & 0,511 \\
\hline 59 & TNRC6A* & 0,493 & RASSF2 & 0,841 & CDKN1A & 2,033 & LRRC32 & 1,1 & CCDC28B & 0,514 \\
\hline 60 & FNBP1 & 0,494 & HLA-E & 0,843 & FAM100B* & 2,059 & NA & 1,101 & HLA-DMB & 0,517 \\
\hline 61 & $\mathrm{ClZ1}$ & 0,495 & BIRC3 & 0,845 & MDFIC & 2,091 & MARCKS & 1,108 & CUX2 & 0,519 \\
\hline 62 & TOMM40 & 0,495 & ANKRD33B* & 0,847 & IER2 & 2,137 & DUSP2 & 1,134 & DUSP22* & 0,523 \\
\hline 63 & TBC1D24* & 0,498 & ANXA7 & 0,85 & DUSP10 & 2,144 & NUDT4 & 1,139 & RPS6KA1 & 0,527 \\
\hline 64 & ILF3 & 0,498 & HVCN1* & 0,85 & FAM102A & 2,198 & CXCR5 & 1,155 & HLA-DOA* & 0,527 \\
\hline 65 & NFKB2 & 0,501 & EPB41L4B* & 0,86 & RAB8B* & 2,198 & IER5 & 1,155 & SYNCRIP & 0,528 \\
\hline 66 & TFDP1* & 0,505 & PIK3CD & 0,864 & PTGER4 & 2,204 & $\mathrm{BCL3}$ & 1,16 & ZNF385C* & 0,533 \\
\hline 67 & ZNF385C* & 0,508 & LAT2 & 0,871 & HLA-DQB1 & 2,206 & SAMSN1 & 1,18 & DENND4A & 0,535 \\
\hline 68 & LYAR* & 0,508 & HCP5 & 0,884 & ARAP2 & 2,214 & NFKBIZ* & 1,2 & PDLIM3* & 0,535 \\
\hline 69 & BIRC3 & 0,508 & HLA-DOA* & 0,894 & SLC7A11 & 2,215 & IL2RA & 1,211 & FYTTD1* & 0,536 \\
\hline 70 & LRP8 & 0,513 & SYNGR2 & 0,901 & MIR155HG* & 2,249 & PARP9* & 1,212 & ELL2* & 0,537 \\
\hline 71 & CCDC28B & 0,514 & ALCAM & 0,905 & CD69 & 2,278 & USP18 & 1,229 & HLA-E & 0,539 \\
\hline 72 & C8orf12* & 0,514 & WNT5A & 0,916 & TSC22D3 & 2,287 & MAP3K8 & 1,333 & IRF2BP2* & 0,539 \\
\hline 73 & HLA-B & 0,519 & RUNX3 & 0,932 & RGS16 & 2,316 & $\mathrm{ZC} 3 \mathrm{H} 12 \mathrm{~A}$ & 1,38 & DNAJB1 & 0,54 \\
\hline 74 & HSP90B1 & 0,523 & BMP2K* & 0,935 & ZFP36L1 & 2,331 & NFKBIA & 1,435 & IGLL1 & 0,545 \\
\hline 75 & KLHDC5* & 0,525 & $\mathrm{CTSH}$ & 0,935 & TRIB3 & 2,354 & RGS16 & 1,437 & TFDP ${ }^{*}{ }^{*}$ & 0,558 \\
\hline 76 & RP9* & 0,526 & PLEKHO1 & 0,94 & IL21R* & 2,373 & GADD45B & 1,481 & PSPC1* & 0,56 \\
\hline 77 & PEA15 & 0,528 & RPS6KA1 & 0,956 & KLF6* & 2,383 & MIR155HG* & 1,498 & ANKRD33B* & 0,562 \\
\hline 78 & MT1X & 0,534 & DUSP22 & 0,963 & PHACTR1 & 2,423 & LINC00158* & 1,525 & CREBZF & 0,565 \\
\hline 79 & MAN1A1 & 0,539 & $\mathrm{IFIH1}$ & 0,991 & SESN2* & 2,446 & PTGER4 & 1,563 & IRF4 & 0,575 \\
\hline 80 & DNAJB1 & 0,543 & OLFML2A & 0,994 & TNFAIP3 & 2,612 & DTX3L* & 1,58 & C8orf12* & 0,579 \\
\hline 81 & SOGA1* & 0,554 & HLA-DQB1 & 0,994 & CD1C & 2,662 & CXCL10 & 1,616 & FNBP1 & 0,617 \\
\hline 82 & CENPV* & 0,555 & COL1A1* & 1,003 & PLEK & 2,716 & TNFAIP3 & 1,636 & ANXA7 & 0,641 \\
\hline 83 & ICAM1 & 0,567 & ICAM1 & 1,006 & FAM46C* & 2,926 & $M \times 1$ & 1,638 & MT1X & 0,67 \\
\hline 84 & CD58 & 0,568 & NFKB2 & 1,009 & CD83 & 2,973 & STAT1 & 1,659 & BATF & 0,702 \\
\hline 85 & NCOR2 & 0,578 & NEIL2* & 1,021 & DDIT3 & 2,977 & BIRC3 & 1,66 & CALR & 0,703 \\
\hline 86 & SNHG12* & 0,585 & FNBP1 & 1,041 & DUSP2 & 3,004 & EGR2 & 1,69 & HLA-DQA ${ }^{*}$ & 0,706 \\
\hline 87 & SLC23A2 & 0,586 & BATF & 1,061 & SQSTM1 & 3,036 & NFKBIE & 1,765 & OLFML2A & 0,714 \\
\hline 88 & OLFML2A & 0,588 & CCDC28B & 1,066 & PHLDA1 & 3,048 & IFIT5 & 1,913 & UMODL1* & 0,716 \\
\hline 89 & LRRFIP1 & 0,59 & MAN1A1 & 1,082 & BHLHE40 & 3,141 & $\mathrm{BCL} 2 \mathrm{~A} 1$ & 1,942 & HLA-DQB1 & 0,723 \\
\hline 90 & DENND4A & 0,599 & CUX2 & 1,089 & SLAMF7* $^{*}$ & 3,147 & IRF1 & 1,965 & IKBKE & 0,723 \\
\hline 91 & IGLL1 & 0,614 & HLA-DQA1* & 1,092 & CCR7 & 3,572 & SLC30A1 & 1,969 & NEIL2* & 0,726 \\
\hline 92 & OVOS2* & 0,63 & DENND4A & 1,101 & $N A^{*}$ & 3,693 & IFIT3* & 1,973 & RILPL2* & 0,779 \\
\hline
\end{tabular}


Table 3 Most prominent altered genes after in vitro interventions of human transformed germinal centre B cells investigated in gene expression profiles of primary samples of aggressive Non-Hodgkin Lymphoma (Continued)

\begin{tabular}{lllllllllll}
\hline $\mathbf{9 3}$ & C1 orf63 & 0,649 & HLA-DPA1 & 1,105 & LINC00158* $^{*}$ & 3,798 & IRF4 & 2,167 & MAN1A1 & 0,781 \\
\hline $\mathbf{9 4}$ & EIF5B & 0,681 & RILPL2 & 1,117 & LY9* $^{*}$ & 4,131 & IFIT1 & 2,186 & CREM & 0,795 \\
\hline $\mathbf{9 5}$ & RUNX3 & 0,702 & DOCK10 & 1,202 & EGR1 $^{*}$ & 4,232 & CMPK2* $^{*}$ & 2,253 & HLA-DPA1 & 0,816 \\
\hline $\mathbf{9 6}$ & NA & 0,704 & BTN2A2 & 1,254 & BCL2A1 & 4,295 & BATF & 2,3 & ACTR3* & 0,833 \\
\hline $\mathbf{9 7}$ & HLA-DQB1 & 0,726 & NA & 1,262 & EGR2 & 4,518 & CD83 & 2,705 & CD58 & 0,834 \\
\hline $\mathbf{9 8}$ & HLA-DQA1 & 0,731 & ELL2* & 1,467 & SGK1 & 4,84 & SGK1 & 2,798 & NA & \\
\hline $\mathbf{9 9}$ & TCOF1 & 0,744 & CD58 & 1,62 & DUSP5 & 5,011 & ICAM1 & 2,918 & LINC00158* & 0,954 \\
\hline $\mathbf{1 0 0}$ & ELL2 $^{*}$ & 0,747 & LINC00158* & 1,793 & RGS1 & 5,476 & IRF9 & 3,155 & IFIT3* & 1,051 \\
\hline
\end{tabular}

Two independent patients cohort were tested. The gene expression from 219 primary lymphoma samples described by Hummel et al. (MMML-1 cohort) and 99 primary lymphoma samples published by Dave et al. (LLMPP-cohort) were compared to global gene expression changes described within the text [20,21]. The deviation from the TOP100 LIMMA gene lists from in vitro interventions is determined by the absence of hybridization probes on the microarrays used for lymphoma gene expression description. In addition multiple hybridization probes for one gene were condensed into a single event.

lymphomas are depicted characterized by a high expression of genes reflecting an increased expression of genes building the $\alpha \operatorname{IgM}$ gene module. Lymphoma cases represented on the left side of the heatmap are characterized by gene expression comparable to unstimulated cells in vitro (BL2). Note that the genes are coherently expressed across lymphoma. There is a continuous gradient when lymphomas are arranged by increasing expression of genes from the $\alpha \operatorname{IgM}$ gene module. Thus, the global gene expression change is absent or present in individual lymphomas
(Figure 4A). Most BLs are characterized by the absence or low expression of the $\alpha \operatorname{IgM}$ gene module and thus lack corresponding pathway activities. This is also observed in the LLMPP cohort (Figure 4B) [21]. Therefore, it is reasonable to believe that individual lymphomas with a high gene module expression are characterized by a stronger activation of oncogenic pathways than those with a low expression of same genes. Therefore human transformed GC B cells (BL2) can be defined as a suitable in vitro model used as surrogate for pathway activity.

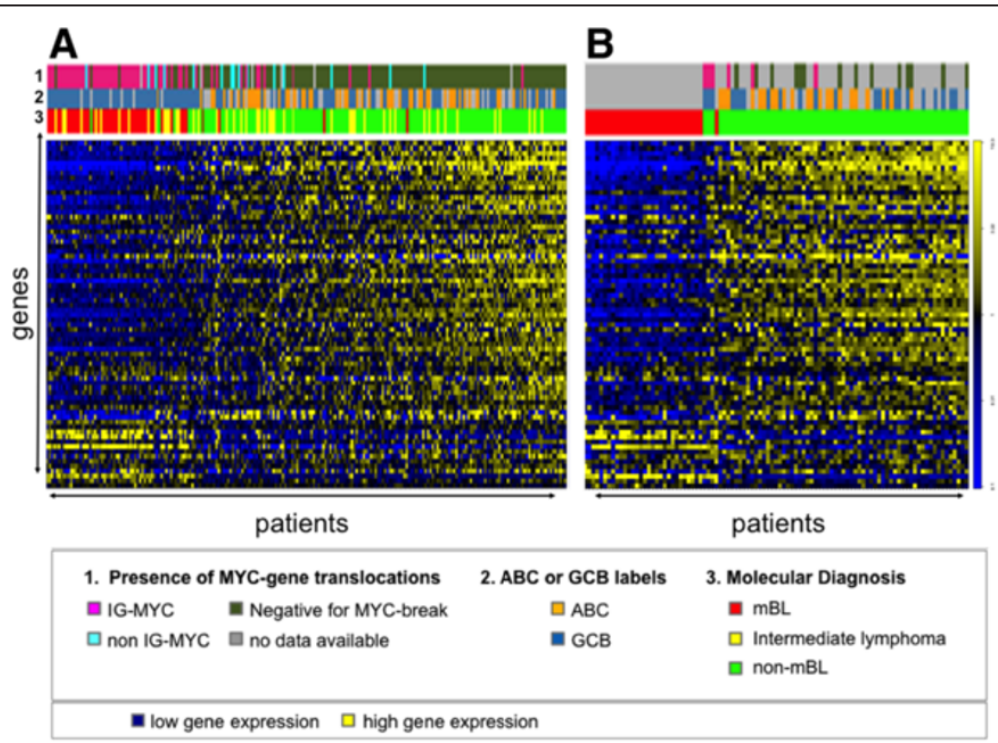

Figure $\mathbf{4}$ Changes in global gene expression separates Burkitt lymphoma and diffuse large B cell lymphoma. The expression of the TOP100 most prominent responding genes upon stimulation of BL2 with algM F(ab) 2 fragments was investigated in primary lymphoma. Left panel: 220 lymphoma cases [20]; right panel: 99 lymphoma cases [21]. NHL cases were ordered from left to right according to their expression of algM affected genes in BL2 cells. The Heatmaps display the expression of target genes (columns) across lymphoma samples (rows). The lower colour bar above the heatmaps marks mBL in red, non-mBL in green and intermediate lymphoma in yellow (molecular diagnosis). The affiliation of samples to $A B C / G C B$ DLBCL subgroups and the presence of an IG-MYC translocation is encoded in the middle and upper bar on top of the map (see legend for colour coding). Relative gene expression is encoded with yellow (high expression) and blue (low expression). As different microarrays were used for the whole genome expression analyses of cell perturbation and patient samples (Affymetrix HGU-133A and HGU133 plus2.0), the list of TOP100 genes had to be adapted to be able to transfer the resulting genes to patient data (see also Table 3, Material and Methods section and Supplementary material for additional details). 


\section{Gene modules of IL21, CD40L or algM is almost perfectly discriminate individual DLBCL}

As BLs are discriminated on the molecular level from other lymphomas as shown by us and Dave et al. [20,21], we next focused on gene expression changes mediated by BAFF, LPS, IL21 or CD40L in vitro in comparison to $\alpha I g M$ in individual DLBCLs (Figure 5). DLBCL cases were arranged according to the activity of the $\alpha \mathrm{IgM}$ gene module. The genes are coherently expressed across lymphomas and there is a continuous gradient when lymphomas are arranged by their increase in the expression of genes from the gene module of IL21 or CD40L in a comparable way as $\alpha \mathrm{IgM}$. This holds also true for the BAFF/LPS driven gene modules within the MMML1 cohort. This highly significant difference is observed by comparing lymphoma cases from the MMML-1 cohort by describing three main groups with low, intermediate and high module activation using corresponding box plots (Additional file 23: Figure S2). The differences are highly significant with respective p-values: $\mathrm{p}<2.2 \mathrm{e}-16 / \mathrm{p}=1.669 \mathrm{e}-10(\alpha \operatorname{IgM})$, $\mathrm{p}<2.2 \mathrm{e}-16 / \mathrm{p}=9.1 \mathrm{e}-07$ (CD40L), $\mathrm{p}<2.2 \mathrm{e}-16 / \mathrm{p}=5.9 \mathrm{e}-08$ (IL21), $\mathrm{p}<2.2 \mathrm{e}-16 / \mathrm{p}=2.614 \mathrm{e}-05$ (BAFF), $\mathrm{p}<2.2 \mathrm{e}-16 /$ $\mathrm{p}=1.6 \mathrm{e}-4$ (LPS) in MMML or LLMPP samples.

The comparison of our data with the recently defined groups of ABC-like or GCB-like DLBCLs reveals no direct association with one of the gene modules presented here (Figure 5, labels on top of the heatmaps) [22]. At the same time, DLBCLs with a MYC translocation are characterized by low gene module activation. Lymphomas carrying a $M Y C$ break are absent in those patients characterized by a higher activation of gene modules. Importantly, DLBCLs characterized by a very high gene module activation show evidence for the expression of genes involved in cell-cell communication or immune responses as well as negative feedback regulatory loops as RGSs and DUSPs [56,57]. A different expression of genes involved in cell-cell communication or immune responses in GCB-like DLBCLs may suggest a different capacity of lymphoma cells to evade immune responses of the host. Furthermore, the activation of negative feedback loops suggests, that although gene modules are typical for acutely activated genes, their outcome seems to be a balance of activating and suppressing signals. These signals imply strong oncogenic pathway activation but also damped cellular activity due to diverse negative feedback reactions or still present tumor suppressor activities.

Highly activated CD58 is part of gene expression changes defined by four stimuli and may present an important marker for DLBCLs. This is in line with recent observations from transcriptome sequencing of DLBCLs. A significant number of DLBCL mutations were identified affecting the CD58 gene [58]. It was suggested that these mutations might play a role in the escape from immune-surveillance of these lymphomas [58,59]. Therefore, it is tempting to speculate that DLBCL with high CD58 expression would be less efficient in immune escape compared to those with reduced CD58 expression or loss of expression due to genetic alterations in this gene. This is also in agreement with our GO analysis, suggesting strong effects on antigen presentation. This is further supported by the expression changes of HLA molecules.

The DUSP family is a set of molecular control molecules which modulate MAPK signalling. DUSPs are affected by all stimuli and also present in the gene modules identified. Their role, either as phosphatases or scaffold proteins, remains to be elucidated as they are involved in defining the magnitude of pathway activity in DLBCLs. The same holds true for the SLAMFs. They play an essential and non-redundant role in the control of humoral immune responses. It would be interesting to investigate whether their expression is functionally linked to the recently observed aberrations in CD58 or $\beta 2 M$ in DLBCLs that might be involved in differences in the capacity to escape host immune responses $[41,58]$.

RGS1 gene expression is characteristic for GCB-like DLBCLs [20]. It is part of the $\alpha$ IgM driven gene module. RGS1 affects chemokine receptor signalling contributing to its desensitization [56]. However, the role of chemokine signalling in lymphomagenesis is not yet fully understood. There are reports suggesting that NHLs express functional chemokine receptors. These, at least in part, dictate tissue localisation and perhaps metastatic potential. However, other reports show that DLBCLs are less sensitive for the CXCR4 ligands CXCL12 and 13 $[60,61]$. The gene expression changes described above for CCR7 and CXCL10 suggest a strong difference of DLBCLs regarding migratory potential and recruitment capacity of cells of the microenvironment but also specific chemokine responsiveness. Because CCR7 and CXCL10 play a pivotal role in the homing of tumour cells as shown by its role in chronic lymphatic leukemia or Hodgkin lymphoma this has to be investigated in the future in more detail. It would be interesting to estimate its role in differences in lymphoma dissemination in relation to the clinical outcome $[43,44,62,63]$.

Strikingly, gene modules of IL21, CD40L or $\alpha \operatorname{IgM}$, even though derived from different data sets, almost perfectly discriminate individual DLBCL. The higher a lymphoma expresses direct $\alpha$ IgM targets the higher it also expresses IL21 or CD40L inducible genes and vice versa. While some explanations can be taken into account, we would favour the following: the aperture of global gene expression changes obtained by computational biology is condensing pathway activities and supports the idea of parallel or equivalent functioning oncogenic activities in individual DLBCLs. 


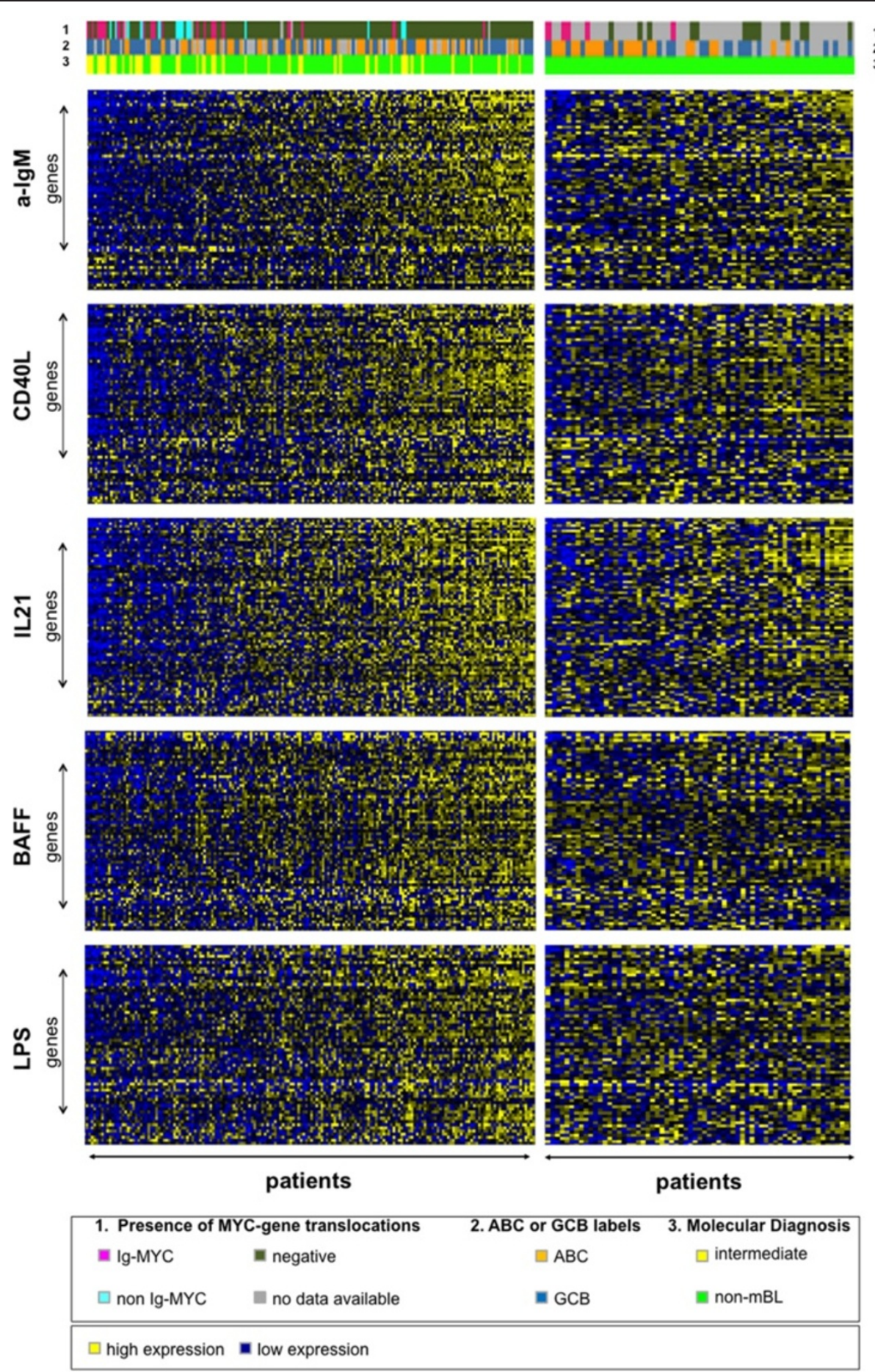

Figure 5 Individual diffuse large B cell lymphomas are characterized by a specific activation of gene modules. The TOP100 most prominent responding genes upon stimulation of BL2 with algM, CD40L, IL21, LPS and BAFF (see also Table 1) were investigated for their expression in the gene expression profiles of two distinct datasets of primary lymphoma. (A) left panel: 175 DLBCLs [20]; right panel: 99 DLBCL cases [21]. DLBCL cases were ordered from left to right according to the similarity of gene expression to the stimulated status of BL2 cells. The Heatmaps display the expression of target genes (columns) across lymphoma samples (rows). The colour bar above the heatmaps marks mBL in red, non-mBL in green and intermediate lymphoma in yellow. The affiliation of samples to ABC/GCB DLBCL subgroups and the presence of an IG-MYC translocation in encoded in a bar on top of the map (see legend for colour coding). Relative gene expression is encoded with yellow (high expression) and blue (low expression). Additional details on statistical significant differences are summarized within Additional file 7: Figure S1. 
We wanted to further explore potential regulatory mechanisms driving differential expression of gene modules. In order to define potential key molecular determinants, signalling pathways involved in the regulation of a set of genes affected by in vitro interventions were specially inhibited using chemical inhibitors.

\section{$B$ cell receptor regulated genes are dominantly affected by ERK1/2 and PI3K activation}

Pathway activation by IL21, CD40L, $\alpha$ IgM, BAFF or LPS reflects qualitative and quantitative differences mediated by the activation of the following pathways: Jak/STAT, NF-кB, JNK1/2, p38a, PI3K, Erk1/2 and $\mathrm{Ca}^{2+}$ influx by immunoblotting, kinase activity measurement or flow cytometry (Additional file 24: Figure S3). We summarized the pathways activated in our model system in a scheme on Figure 6A. $\alpha \operatorname{IgM}$ treatment is associated with $\mathrm{Ca}^{2+}$ mobilization. Furthermore Erk1/2, Akt and p38a phosphorylation or enhanced activity of JNK is observed. In addition, the canonical and non-canonical NFKB pathways are activated to some extent as revealed by IKB $\alpha$ degradation and p100 to p52 processing. CD40L activates both canonical and non-canonical NF-KB at the highest level compared to the other stimuli. In addition a p38 phosphorylation and JNK kinase activity is observed comparable to that of $\alpha \operatorname{IgM}$ treatment. IL21 stimulation of BL2 cells is mainly associated with STAT1 and STAT3 activation as shown by tyrosine phosphorylation. A slightly reduced expression of ІкB $\alpha$ after IL21 treatment is observed, suggesting an activation of the canonical NF-кB. Thus, the perfect discrimination of individual DLBCLs by three different gene modules suggest different magnitudes of simultaneous oncogenic activities mediated by for example Jak/STAT, NF-кB, MAPK (MAPK8/JNK1, MAPK14/p38a, MAP2K1/2), PI3K and $\mathrm{Ca}^{2+}$ mediated responses.

Of the stimuli used in this study, $\alpha \operatorname{IgM}$ treatment had the strongest effects on gene expression in vitro and was capable to activate a wide range of signalling pathways. Therefore, we wanted to further explore pathways involved in the observed differences between individual lymphomas characterized by specific gene module activation. We used chemical kinase inhibitors to identify the pathways involved in the regulation of gene modules in response to stimulation. The utilized inhibitors are summarized in a scheme in Figure $6 \mathrm{~B}$ showing the hierarchy of kinases in a prior knowledge scheme [2]. The following kinases were considered: MAPK including $\mathrm{p} 38, \mathrm{JNK} 1 / 2$ or MAP2K1/2 affecting Erk1/2 activation or MAP3K7/TAK1 potentially involved in NF- $\mathrm{B}$ and MAPK signalling. Furthermore, we investigated IKK2 as part of NF-KB signalling and PI3K as it is involved in numerous pathways activated through $\alpha \operatorname{IgM}$, including Akt.
BL2 cell were preincubated for 3 hrs with specific inhibitors and then stimulated by $\alpha \operatorname{IgM}$ for additional $3 \mathrm{hrs}$ in the presence of respective inhibitors.

The expression of SGK1, PYGO1, SLAMF3, DUSP10, EGR2, ID3, CCR7, DUSP2, SLAMF6, BCL6, MYC, LEF1, BCL9, IRF4 and RGS1, DUSP5, SLAMF7 after $\alpha \operatorname{IgM}$ treatment was investigated in the absence or presence of the above mentioned kinase inhibitors. Three main groups of regulatory interactions are observed:

Within the first group are genes affected by U0126 interrupting the activity of MAP2K1/2 and Ly294002 inhibiting PI3K. Within this group are SGK1, PYGO1, SLAMF3/7 and DUSP10 or BCL6, (Figure 6C, D, E and Additional file 25: Figure S4 A, B, D). This suggests a central role for Erk1/2 and PI3K. Within the second group are genes, dominantly affected by U0126 but not Ly294002. The expression of EGR2, ID3, CCR7, DUSP2/ 5 or SLAMF6 and RGS1 is mostly regulated by Erk $1 / 2$ (Figure 6F, G, H, I, J, N and Additional file 25: Figure S4 E). In addition, a third group of genes including $M Y C, L E F 1$ as well as BCL9 is affected by Ly294002 but not U0126 (Figure 6K, L and Additional file 25: Figure S4C).

Interestingly, IRF4 is the only gene which $\alpha \operatorname{IgM}$ affected gene expression is regulated through TAK1/IKK2/p38 (correspondingly 5Z-7-oxozeaenol / ACHP / SB203580) without Erk1/2 or PI3K involvement (Figure $6 \mathrm{M}$ ).

In addition, $\alpha \operatorname{IgM}$ mediated activation of $S G K 1$ is affected by TAK1 inhibition (Figure 6C), whereas for example CCR7 activation is regulated through TAK1 and JNK (Figure 6J). Furthermore, for SGK1, ID3, CCR7 or SLAMF6, the effect of the TAK-inhibitor is not accompanied by a comparable IKK2 inhibition. Whereas for CCR7 and ID3 the known signalling cascade TAK1-JNK can be proposed, for $S G K 1$ either a more direct TAK1 effect or a PI3K-TAK1-Erk1/2 cascade has to be taken into account (Figure 6C, G, H). Whereas the expression of PYGO1 is affected by the well-known TAK1-IKK2 cascade (Figure 6D, I) for SLAMF6 and IRF4 also the TAK1-p38 cascade seems to play a role (Figure 6J, M).

aIgM mediated $M Y C$ inhibition is reversed by the PI3K inhibitor Ly294002. This demonstrates an involvement of PI3K signalling to inhibit aberrant $M Y C$ expression (Figure 6K). Furthermore, an effect of JNK-, IKK2- or PI3K inhibition on basal expression of $M Y C$ can be observed. This supports a role of a tonic activation (basal signalling in unstimulated BL2 cells) of PI3K, JNK and IKK2 mediated signalling activity in regulating aberrant "basal" MYC expression. Interestingly, a new murine model for lymphomas has been described supporting the view of a synergistic action of c-Myc and PI3K signalling [64]. Furthermore, a tonic BCR signalling and PI(3) kinase activity in Burkitt's lymphoma has been recently described by Schmitz and co-workers [65]. However, this link between tonic PI3K signalling and $M Y C$ expression 


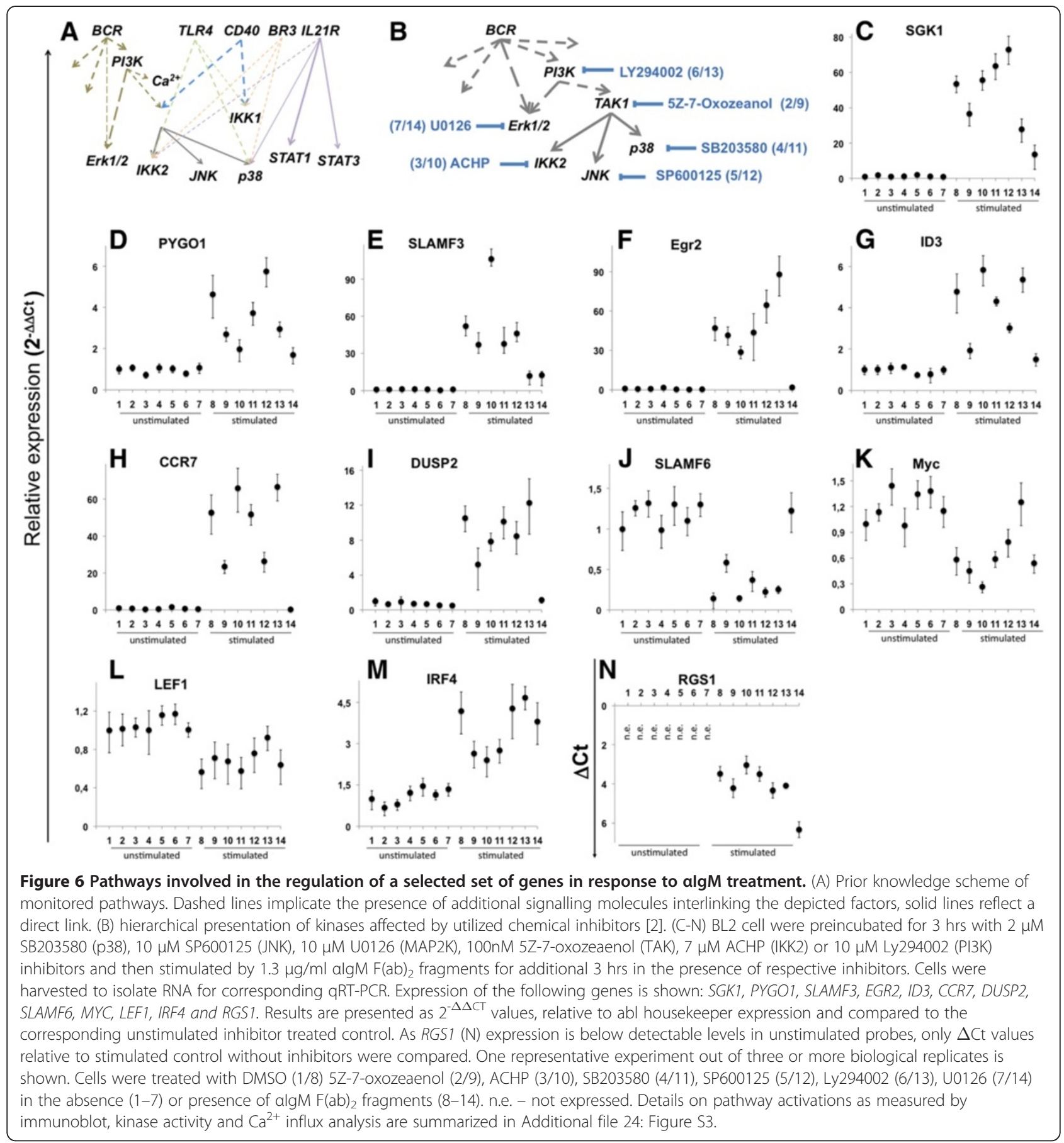

has not been described in this publication. Interestingly, in this study treatment of BL lines with BKM120, a PI(3) kinase inhibitor in clinical trials, or rapamycin, an inhibitor of the mTORC1 complex, was toxic to most BL lines after 4 days. Therefore, their rapamycin signature has to be taken into account for future investigations. Surprisingly, IKK2 inhibition was associated with a much stronger $\alpha \operatorname{IgM}$ mediated suppression of $M Y C$ expression (Figure 6K) [66]. Therefore, we observed a suppressive role of tonic IKK2 activity onto MYC expression in BL2 cells. This sheds new light onto the regulation of the aberrant expression of $M Y C$. Positive and negative signals from PI3K, MAPK and NF-kB pathways can now be investigated in more detail for example in order to delineate differences between BLs and DLBCLs characterized by a high Myc-index or $M Y C$ break [67].

A comparable effect of PI3K-inhibition as described for $M Y C$ is observed also for BCL6, LEF1 and BCL9 
(Figure 6L and Additional file 24: Figure S3 B, C). However, as for $M Y C$, the expression of BCL6 or BCL9 is already affected to some extend by Ly294002 in unstimulated BL2 cells. Therefore, it is difficult to interpret these data for BCL6 and BCL9 to the end (Additional file 25: Figure $\mathrm{S} 4 \mathrm{~B}, \mathrm{C})$. We speculate that combinations of pathways are involved in both basal and $\alpha$ IgM mediated gene expression.

In Figure 7A a scheme summarizes the main effects of kinase inhibition observed after $\alpha$ IgM treatment.

As already noted above, in some cases the treatment of cells with inhibitors is associated with an enhanced activation or inhibition of respective genes. For example treatment of cells with Ly294002 led to a stronger activation of EGR2 or CCR7 by $\alpha \mathrm{IgM}$ treatment (Figure 6E, G). Comparable effects are observed for IKK2 inhibition for SLAMF3 and ID3, for p38 or JNK inhibition analysing $S G K 1$, ID3 or PYGO1 respectively (Figure $6 \mathrm{C}, \mathrm{D}, \mathrm{E}, \mathrm{G})$. In Figure $7 \mathrm{~B}$ a respective summary of main $\alpha \operatorname{IgM}$ enhancing effects after inhibition of specific kinases is shown, including effects of these inhibitors onto the basal expression levels of analysed genes as for example $M Y C$ or BCL9.

Overall, we found that the expression of most of the analyzed genes affected by $\alpha \operatorname{IgM}$ treatment is regulated through Erk1/2 activation accompanied by PI3K, TAK1 and partially to lower extent by IKK2 and JNK.

Erk and PI3K signalling is exclusive to the $\alpha I g M$ gene module (Additional file 24: Figure S3 D, G). These pathways are not affected by the other in vitro treatments Activated NF-KB signalling seems to be less important for the $\alpha \operatorname{IgM}$ gene module. However, the analysis of CD40 mediated expression of ICAM1, CD58, SLAMF3 or CCR7 revealed a strong involvement of NF-кB signalling (data not shown). Our analysis supports the idea that the MAPK/Erk-pathway has a major impact on gene expression in individual DLBCL with a high activation of the $\alpha \operatorname{IgM}$ gene module. Therefore, it is reasonable to discuss the use of drugs targeting Erk $1 / 2$ for a subgroup of DLBCL characterized by a high activation of the $\alpha \operatorname{IgM}$ driven gene module [68]. In a recent study, a molecular interaction of Erk and CHK2 was shown to affect DNA-damage response and apoptosis of DLBCLs [69]. The recently described success of using Syk or Btk inhibitors or even mTOR and PKC inhibitors to treat DLBCL might be explained by the activity of these signalling pathways [70-75]. We are aware of the limitations of chemical kinase inhibitors to analyse pathway elements. However, as comparable compounds are developed for clinical applications, the information drawn from studies integrating in vitro stimulations as pathway surrogates with gene expression of individual lymphoma patients will provide comprehensive insights into potential targets for therapy. In the future the utilized in vitro stimulations can be used in combination with kinase inhibitors to delineate respective pathway interactions as for example a link between TAK1 and Erk1/2 or the different branches within PI3K signalling

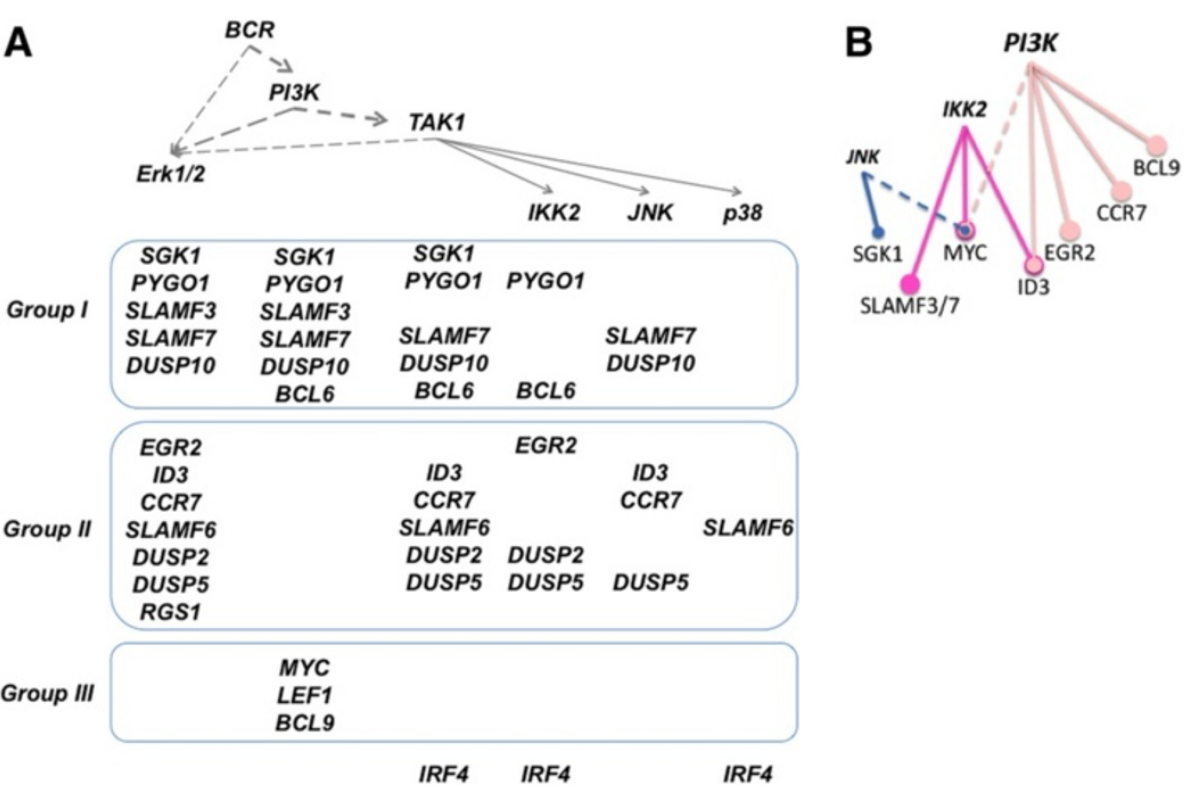

Figure 7 Schemes summarizing investigated kinase involvement in algM mediated gene expression. algM mediated activation / induction of gene expression (A) and suppressive effects of pathway elements onto selected genes affecting basal and algM mediated gene expression (B). Within the scheme (A) genes are listed and sorted according to the groups described within the main text in relation to corresponding kinases. Data for gene expression changes of BCL6, BCL9, DUSP5, DUSP10 and SLAMF7 are described within Additional file 25: Figure S4. 
by applying also alternative experimental approaches. Furthermore, our data indicate that a global investigation of kinase inhibitors and their combinations would be useful for a better understanding of gene regulation of global gene expression changes and their integration with patient's data.

\section{Conclusions}

We provide an in vitro model system to investigate pathway activations qualitatively and quantitatively. B cell specific stimuli are used to identify gene expression changes allowing to "switch" gene expression from one steady state level characteristic for BL towards that of DLBCLs. We defined the extent to which specific signalling pathways are responsible for differences in gene expression that distinguish individual DLBCL. Gene modules of IL21, CD40L or $\alpha$ IgM discriminate individual DLBCL, from each other, even though derived from different data sets. The greater an individual lymphoma expresses $\alpha \operatorname{IgM}$ target genes, the greater it will also express IL21 or CD40L regulated genes.

We have shown that mitogen activated protein kinaseand phosphoinositide 3 kinase-signaling are an important part of pathway networks describing differences in gene expression that distinguish individual DLBCL. This observation supports recent findings about the role of tonic and/or chronic active MAPK signalling in individual lymphoma and might therefore constitute a promising target for future therapy approaches. Although the discrimination of individual DLBCL by three different gene modules suggest different magnitudes of parallel or equivalent oncogenic activities mediated by Jak/STAT, NF-кВ, MAPK. Therefore, transformed human germinal centre B cells can be used to test new compounds and their influence on the respective pathways in DLBCLs. A useful tool to test for individual treatment strategies is offered, which is independent from heterogeneous lymphoma associated mutations know from DLBCLs.

\section{Materials and methods}

\section{Cell culture and stimulation}

BL2 cells were cultivated as described previously at cell densities between $2 \times 10^{5}$ and $1 \times 10^{6}$ cells $/ \mathrm{ml}$ [63]. For stimulation studies, cells were cultured in cell culture medium supplemented with $10 \mathrm{mM}$ HEPES at $1 \times 10^{6}$ cells $/ \mathrm{ml}$ and incubated with indicated reagents for up to 9 hrs. To crosslink the BCR, BL2 cells were cultured in the presence of $1.3 \mu \mathrm{g} / \mathrm{ml}$ goat $\alpha \operatorname{IgM~} \mathrm{F}(\mathrm{ab})_{2}$ fragments (Jackson Immunity). Recombinant human sCD40L (AutogenBioclear), human BAFF (R\&D Systems) and recombinant human IL21 (Peprotech) were used at a concentration of $200 \mathrm{ng} / \mathrm{ml}, 100 \mathrm{ng} / \mathrm{ml}$ and $100 \mathrm{ng} / \mathrm{ml}$ respectively. LPS (E. coli strain 055:B5, Sigma) was added to the cells at a concentration of $1 \mu \mathrm{M}$.
Cells were harvested using corresponding inhibitors of phosphatases and proteases and RNA was isolated using the RNeasy Plus Mini Kit (Qiagen).

Immunoblot, Calcium Measurement, JNK Immunocomplex kinase assays and qRT-PCR analysis are summarized within supplemental Material and Methods.

\section{Gene expression analysis}

For gene expression analysis RNA was isolated with RNeasy Plus Mini Kit (Qiagen) according to the manufacturer's instructions. For real time PCR analysis RNA was reverse transcribed using SuperScript II Reverse Transcriptase (Invitrogen) and random hexamer primers (IBA BioTAGnology). cDNA samples were further analysed by SYBR Green-based real-time PCR using the 7900HT Fast Real-Time PCR System (Applied Biosystems) (additional details for used primers within the Additional file 26: Table S17). For whole genome micorarrays RNA was labelled for microarray hybridization using Affymetrix GeneChip ${ }^{\circledR}$ IVT Labelling Kit (Affymetrix). Fragmentation and hybridization of labelled anti sense RNA on Human Genome U133A 2.0 plus Arrays (Affymetrix) was performed according to manufacturer's recommendations by the Kompetenzzentrum für Fluoreszente Bioanalytik. Rawdata have been uploaded to GEO and can be assessed using GSE42660. Gene expression values were obtained by first correcting for the background and normalizing on probe level using the variance stabilization method by Huber and colleagues [55]. The normalized probe intensities were summarized into gene expression levels by using an additive model fitted by the median polish procedure [76,77]. If there was more than one probeset per gene, we kept the probeset best responding. This was done by looking at the fold changes between control and stimulation, the probeset with the highest fold change was kept. Additional details for Biostatistics are summarized within supplemental Material and Methods. Ethical approval for gene expression studies on human lymphoma material was granted and described in detail by Hummel and colleagues [20] as well as Dave and colleagues [21]. These studies were conducted in compliance with the Declaration of Helsinki.

\section{Additional files}

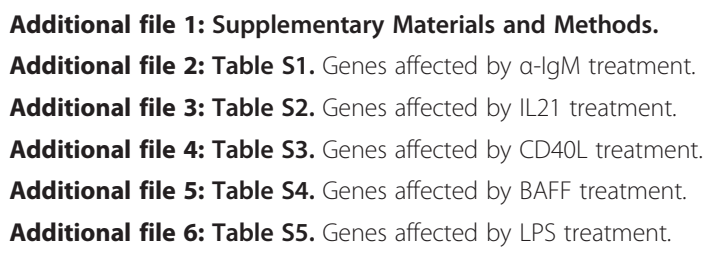

Additional file 7: Figure S1. Global gene expression changes of CD40L stimulation are highly comparable in distinct Burkitt Lymphoma cell lines (Ramos and BL2). 
Additional file 8: Supplemental 1. A selection of microarray data providing insight into gene expression changes affected by CD40L, B cell receptor activation, for BAFF, LPS or IL21

Additional file 9: Supplemental 2. Geneset enrichment Analysis identifying enriched pathways in differentially expressed genes.

Additional file 10: Table S6. Differentially expressed genes overlapping between CD40L and IL21 stimulation.

Additional file 11: Table S7. Differentially expressed genes overlapping between a-IgM and IL21 stimulation.

Additional file 12: Table S8. Differentially expressed genes overlapping between LPS and IL21 stimulation.

Additional file 13: Table S9. Differentially expressed genes overlapping between BAFF and IL21 stimulation.

Additional file 14: Table S10. Differentially expressed genes overlapping between CD40L and a-lgM stimulation.

Additional file 15: Table S11. Differentially expressed genes overlapping between CD40L and LPS stimulation.

Additional file 16: Table S12. Differentially expressed genes overlapping between CD40L and BAFF stimulation.

Additional file 17: Table S13. Differentially expressed genes overlapping between LPS and a-IgM stimulation.

Additional file 18: Table S14. Differentially expressed genes overlapping between BAFF and a-IgM stimulation.

Additional file 19: Table S15. Differentially expressed genes overlapping between LPS and BAFF stimulation.

Additional file 20: Supplemental 3. Geneset enrichment Analysis identifying enriched pathways in differentially expressed genes overlapping between stimulations.

Additional file 21: Table S16. Genes differentially expressed in response to only one specific stimulation (unique).

Additional file 22: Supplemental 4. Geneset enrichment Analysis identifying enriched pathways in differentially expressed genes unique for each specific stimulation.

Additional file 23: Figure S2. Lymphoma cases showing a high expression of a-lgM responsive genes as well characterized by a high expression of the genes affected by the other analysed interventions.

Additional file 24: Figure S3. Qualitative and quantitative differences in pathway activation by IL21, CD40L, algM, BAFF or LPS in human transformed GC B cells in vitro.

Additional file 25: Figure S4. Pathways involved in the regulation of a selected set of induced genes in response to algM treatment.

Additional file 26: Table S17. Utilized Oligonucleotides.

\section{Competing interests}

The authors declare that they have no competing interests.

\section{Authors' contributions}

$S A, V M, K A, E M$ performed experiments and wrote the manuscript. VBF, HE, UA, MK, LD performed experiments. MK, performed statistics and wrote the manuscript. HM, TL wrote the manuscript. KD. design of the study, wrote the manuscript and finally approved. All authors read and approved the final manuscript.

\section{Acknowledgement}

This work was supported by grants from the Deutsche Krebshilfe (Network "Molecular mechanisms of malignant lymphoma"), BMBF network on Medical Systems biology HaematoSys $(0315452 \mathrm{H})$ and the Deutsche

Forschungsgemeinschaft (GRK1034, FOR942), the Leukemia and Lymphoma Research Fund (M.V.), the Kubeschka-Stricker-Wirth-Stiftung at the GeorgAugust-Universität (DK) and a DAAD fellowship (A.S.). We thank R. Spang and all other members of the network Medical Systems biology "HaematoSys for helpful discussions throughout all aspects of the manuscript. We are especially grateful to Sarah Leonard and Jennifer Anderton for revising the manuscript.

\section{Author details}

'Department of Haematology and Oncology, University Medical Centre Göttingen, Göttingen, Germany. ${ }^{2}$ Computational Diagnostics Group, Institute for Functional Genomics, University of Regensburg, Regensburg, Germany. ${ }^{3}$ School of Cancer Sciences, University of Birmingham, Birmingham, UK. ${ }^{4}$ Institute for Pathology, Campus Benjamin Franklin Charité Berlin, Berlin, Germany. ${ }^{5}$ Research Unit Gene Vectors, Helmholtz Zentrum München German Research Center for Environmental Health, München, Germany. ${ }^{6}$ Department of Cellular and Molecular Immunology, University Medical Centre Göttingen, Göttingen, Germany. ${ }^{7}$ Network "Molecular Mechanism of Malignant Lymphoma" (MMML) of the Deutsche Krebshilfe, Göttingen, Germany. ${ }^{8}$ FOR942 of the Deutsche Forschungsgemeinschaft at the University Medical Centre Göttingen, Göttingen, Germany. ${ }^{9} \mathrm{GRK} 1034$ of the Deutsche Forschungsgemeinschaft, Göttingen, Germany. ${ }^{10}$ Network HaematoSys, Leipzig, Germany. ${ }^{11}$ Department of Pediatrics I, University Medical Centre Göttingen, Göttingen, Germany. ${ }^{12}$ Zentrum für Innere Medizin, Abteilung Hämatologie und Onkologie, Universitätsmedizin der Georg-August-Universität Göttingen, 37099, Göttingen, Germany.

Received: 4 July 2012 Accepted: 25 November 2012

Published: 20 December 2012

\section{References}

1. Klein U, Dalla-Favera R: Germinal centres: role in B-cell physiology and malignancy. Nat Rev Immunol 2008, 8:22-33.

2. Shaffer AL, Young RM 3rd, Staudt LM: Pathogenesis of human B cell lymphomas. Annu Rev Immunol 2012, 30:565-610.

3. Swerdlow SH, Campo E, Harris NL, et al: WHO Classification of Tumours of Haematopoietic and Lymphoid Tissues. In Atlas of Hematologic Neoplasms. 4th edition. France: IARC Press; 2008.

4. Coiffier B: State-of-the-art therapeutics: diffuse large B-cell lymphoma. J Clin Oncol 2005, 23:6387-6393.

5. Pfreundschuh $M$, Schubert J, Ziepert M, Schmits R, Mohren M, Lengfelder E, Reiser M, Nickenig C, Clemens M, Peter N, et al: Six versus eight cycles of bi-weekly CHOP-14 with or without rituximab in elderly patients with aggressive CD20+ B-cell lymphomas: a randomised controlled trial (RICOVER-60). Lancet Oncol 2008, 9:105-116.

6. Kuppers R, Dalla-Favera R: Mechanisms of chromosomal translocations in B cell lymphomas. Oncogene 2001, 20:5580-5594

7. Tolar P, Sohn HW, Liu W, Pierce SK: The molecular assembly and organization of signaling active B-cell receptor oligomers. Immuno/ Rev 2009, 232:34-41.

8. Chiron D, Bekeredjian-Ding I, Pellat-Deceunynck C, Bataille R, Jego G: Toll-like receptors: lessons to learn from normal and malignant human B cells. Blood 2008, 112:2205-2213.

9. Bishop GA: The many faces of CD40: multiple roles in normal immunity and disease. Semin Immunol 2009, 21:255-256.

10. Gallagher E, Enzler T, Matsuzawa A, Anzelon-Mills A, Otero D, Holzer R, Janssen E, Gao M, Karin M: Kinase MEKK1 is required for CD40-dependent activation of the kinases Jnk and p38, germinal center formation, B cell proliferation and antibody production. Nat Immunol 2007, 8:57-63.

11. Kim SJ, Lee SJ, Choi IY, Park Y, Choi CW, Kim IS, Yu W, Hwang HS, Kim BS: Serum BAFF predicts prognosis better than APRIL in diffuse large B-cell lymphoma patients treated with rituximab plus CHOP chemotherapy. Eur J Haematol 2008, 81:177-184.

12. Zeng R, Spolski R, Casas E, Zhu W, Levy DE, Leonard WJ: The molecular basis of IL-21-mediated proliferation. Blood 2007, 109:4135-4142.

13. Davis RE, Ngo VN, Lenz G, Tolar P, Young RM, Romesser PB, Kohlhammer H, Lamy L, Zhao H, Yang Y, et al: Chronic active B-cell-receptor signalling in diffuse large B-cell lymphoma. Nature 2010, 463:88-92.

14. Engelke $\mathrm{M}$, Engels $\mathrm{N}$, Dittmann $\mathrm{K}$, Stork $\mathrm{B}$, Wienands J: $\mathrm{Ca}(2+)$ signaling in antigen receptor-activated B lymphocytes. Immunol Rev 2007, 218:235-246.

15. Khiem D, Cyster JG, Schwarz JJ, Black BL: A p38 MAPK-MEF2C pathway regulates B-cell proliferation. Proc Natl Acad Sci USA 2008, 105:17067-17072

16. Krappmann D, Wegener E, Sunami $Y$, Esen M, Thiel A, Mordmuller B, Scheidereit C: The lkappaB kinase complex and NF-kappaB act as master regulators of lipopolysaccharide-induced gene expression and control subordinate activation of AP-1. Mol Cell Biol 2004, 24:6488-6500. 
17. Compagno M, Lim WK, Grunn A, Nandula SV, Brahmachary M, Shen Q, Bertoni F, Ponzoni M, Scandurra M, Califano A, et al: Mutations of multiple genes cause deregulation of NF-kappaB in diffuse large B-cell lymphoma. Nature 2009, 459:717-721.

18. Ngo VN, Young RM, Schmitz R, Jhavar S, Xiao W, Lim KH, Kohlhammer H, Xu W, Yang $Y$, Zhao $H$, et al: Oncogenically active MYD88 mutations in human lymphoma. Nature 2011, 470:7332.

19. Zhu X, Hart R, Chang MS, Kim JW, Lee SY, Cao YA, Mock D, Ke E, Saunders $B$, Alexander A, et al: Analysis of the major patterns of B cell gene expression changes in response to short-term stimulation with 33 single ligands. J Immunol 2004, 173:7141-7149.

20. Hummel M, Bentink S, Berger H, Klapper W, Wessendorf S, Barth TF, Bernd HW, Cogliatti SB, Dierlamm J, Feller AC, et al: A biologic definition of Burkitt's lymphoma from transcriptional and genomic profiling. $N$ Engl J Med 2006, 354:2419-2430.

21. Dave SS, Fu K, Wright GW, Lam LT, Kluin P, Boerma EJ, Greiner TC, Weisenburger DD, Rosenwald A, Ott G, et al: Molecular diagnosis of Burkitt's lymphoma. N Engl J Med 2006, 354:2431-2442.

22. Alizadeh AA, Eisen MB, Davis RE, Ma C, Lossos IS, Rosenwald A, Boldrick JC, Sabet $H$, Tran T, Yu X, et al: Distinct types of diffuse large B-cell lymphoma identified by gene expression profiling. Nature 2000 , 403:503-511.

23. Feuerhake F, Kutok JL, Monti S, Chen W, LaCasce AS, Cattoretti G, Kurtin P, Pinkus GS, de Leval L, Harris NL, et al: NFkappaB activity, function, and target-gene signatures in primary mediastinal large B-cell lymphoma and diffuse large B-cell lymphoma subtypes. Blood 2005, 106:1392-1399.

24. Lenz G, Wright G, Dave SS, Xiao W, Powell J, Zhao H, Xu W, Tan B, Goldschmidt N, lqbal J, et al: Stromal gene signatures in large-B-cell lymphomas. N Engl J Med 2008, 359:2313-2323.

25. Monti S, Savage KJ, Kutok JL, Feuerhake F, Kurtin P, Mihm M, Wu B, Pasqualucci L, Neuberg D, Aguiar RC, et al: Molecular profiling of diffuse large B-cell lymphoma identifies robust subtypes including one characterized by host inflammatory response. Blood 2005, 105:1851-1861.

26. Bentink S, Wessendorf S, Schwaenen C, Rosolowski M, Klapper W, Rosenwald A, Ott G, Banham AH, Berger H, Feller AC, et al: Pathway activation patterns in diffuse large B-cell lymphomas. Leukemia 2008, 22:1746-1754

27. Mori S, Rempel RE, Chang JT, Yao G, Lagoo AS, Potti A, Bild A, Nevins JR: Utilization of pathway signatures to reveal distinct types of $B$ lymphoma in the Emicro-myc model and human diffuse large B-cell lymphoma. Cancer Res 2008, 68:8525-8534.

28. Shaffer AL, Wright G, Yang L, Powell J, Ngo V, Lamy L, Lam LT, Davis RE, Staudt LM: A library of gene expression signatures to illuminate normal and pathological lymphoid biology. Immunol Rev 2006, 210:67-85.

29. Ding BB, Yu JJ, Yu RY, Mendez LM, Shaknovich R, Zhang Y, Cattoretti G, Ye $\mathrm{BH}$ : Constitutively activated STAT3 promotes cell proliferation and survival in the activated B-cell subtype of diffuse large B-cell lymphomas. Blood 2008, 111:1515-1523.

30. Lam LT, Wright G, Davis RE, Lenz G, Farinha P, Dang L, Chan JW, Rosenwald A, Gascoyne RD, Staudt LM: Cooperative signaling through the signal transducer and activator of transcription 3 and nuclear factor-\{kappa\}B pathways in subtypes of diffuse large B-cell lymphoma. Blood 2008, 111:3701-3713.

31. Jazirehi AR, Vega MI, Chatterjee D, Goodglick L, Bonavida B: Inhibition of the Raf-MEK1/2-ERK1/2 signaling pathway, $\mathrm{BCl}-\mathrm{xL}$ down-regulation, and chemosensitization of non-Hodgkin's lymphoma B cells by Rituximab. Cancer Res 2004, 64:7117-7126.

32. Kloo B, Nagel D, Pfeifer M, Grau M, Duwel M, Vincendeau M, Dorken B, Lenz $P$, Lenz G, Krappmann D: Critical role of PI3K signaling for NF-kappaBdependent survival in a subset of activated B-cell-like diffuse large B-cell lymphoma cells. Proc Natl Acad Sci USA 2011, 108:272-277.

33. Denepoux S, Razanajaona D, Blanchard D, Meffre G, Capra JD, Banchereau J, Lebecque $S$ : Induction of somatic mutation in a human B cell line in vitro. Immunity 1997, 6:35-46.

34. Faili A, Aoufouchi S, Gueranger Q, Zober C, Leon A, Bertocci B, Weill JC, Reynaud CA: AID-dependent somatic hypermutation occurs as a DNA single-strand event in the BL2 cell line. Nat Immunol 2002, 3:815-821.

35. Henriquez NV, Floettmann E, Salmon M, Rowe M, Rickinson AB: Differential responses to $C D 40$ ligation among Burkitt lymphoma lines that are uniformly responsive to Epstein-Barr virus latent membrane protein 1. $\mathrm{J}$ Immunol 1999, 162:3298-3307.
36. Smyth GK, Michaud J, Scott HS: Use of within-array replicate spots for assessing differential expression in microarray experiments. Bioinformatics 2005, 21:2067-2075.

37. Basso K, Klein U, Niu H, Stolovitzky GA, Tu Y, Califano A, Cattoretti G, DallaFavera R: Tracking CD40 signaling during germinal center development. Blood 2004, 104:4088-4096.

38. Benjamini $Y$, Hochberg $Y$ : Controlling the false discovery rate: a practical and powerful approach to multiple testing. J R Statist Soc B 1995, 57:289-300

39. Dennig D, Lacerda J, Yan Y, Gasparetto C, O'Reilly RJ: ICAM-1 (CD54) expression on $B$ lymphocytes is associated with their costimulatory function and can be increased by coactivation with IL-1 and IL-7. Cell Immunol 1994, 156:414-423.

40. Zhu DM, Dustin ML, Cairo CW, Thatte HS, Golan DE: Mechanisms of Cellular Avidity Regulation in CD2-CD58-Mediated T Cell Adhesion. ACS Chem Biol 2006, 1:649-658.

41. Schwartzberg PL, Mueller KL, Qi H, Cannons JL: SLAM receptors and SAP influence lymphocyte interactions, development and function. Nat Rev Immunol 2009, 9:39-46.

42. Gatto D, Wood K, Brink R: EBI2 operates independently of but in cooperation with CXCR5 and CCR7 to direct B cell migration and organization in follicles and the germinal center. J Immunol 2011, 187:4621-4628.

43. Rehm A, Mensen A, Schradi K, Gerlach K, Wittstock S, Winter S, Buchner G, Dorken B, Lipp M, Hopken UE: Cooperative function of CCR7 and lymphotoxin in the formation of a lymphoma-permissive niche within murine secondary lymphoid organs. Blood 2011, 118:1020-1033.

44. Teruya-Feldstein J, Jaffe ES, Burd PR, Kanegane H, Kingma DW, Wilson WH, Longo DL, Tosato G: The role of Mig, the monokine induced by interferon-gamma, and IP-10, the interferon-gamma-inducible protein-10, in tissue necrosis and vascular damage associated with Epstein-Barr virus-positive lymphoproliferative disease. Blood 1997, 90:4099-4105

45. Zebedee Z, Hara E: Id proteins in cell cycle control and cellular senescence. Oncogene 2001, 20:8317-8325.

46. Sun $\mathrm{XH}$ : Constitutive expression of the Id 1 gene impairs mouse $\mathrm{B}$ cell development. Cell 1994, 79:893-900.

47. Basso K, Dalla-Favera R: BCL6: master regulator of the germinal center reaction and key oncogene in B cell lymphomagenesis. Adv Immunol 2010, 105:193-210

48. Shaffer AL, Yu X, He Y, Boldrick J, Chan EP, Staudt LM: BCL-6 represses genes that function in lymphocyte differentiation, inflammation, and cell cycle control. Immunity 2000, 13:199-212.

49. Shaffer AL, Emre NC, Romesser PB, Staudt LM: IRF4: Immunity. Malignancy! Therapy? Clin Cancer Res 2009, 15:2954-2961.

50. Shaffer AL, Lin Kl, Kuo TC, Yu X, Hurt EM, Rosenwald A, Giltnane JM, Yang L, Zhao H, Calame K, Staudt LM: Blimp-1 orchestrates plasma cell differentiation by extinguishing the mature $B$ cell gene expression program. Immunity 2002, 17:51-62.

51. Alizadeh AA, Gentles AJ, Alencar AJ, Liu CL, Kohrt HE, Houot R, Goldstein MJ, Zhao S, Natkunam $Y$, Advani RH, et al: Prediction of survival in diffuse large B-cell lymphoma based on the expression of 2 genes reflecting tumor and microenvironment. Blood 2011, 118:1350-1358.

52. Lossos IS, Alizadeh AA, Rajapaksa R, Tibshirani R, Levy R: HGAL is a nove interleukin-4-inducible gene that strongly predicts survival in diffuse large B-cell lymphoma. Blood 2003, 101:433-440.

53. Kim J, Kim DW, Chang W, Choe J, Park CS, Song K, Lee I: Wnt5a is secreted by follicular dendritic cells to protect germinal center B cells via Wnt/ Ca2+/NFAT/NF-kappaB-B cell lymphoma 6 signaling. J Immunol 2012, 188:182-189.

54. Lottaz C, Yang X, Scheid S, Spang R: OrderedList-a bioconductor package for detecting similarity in ordered gene lists. Bioinformatics 2006, 22:2315-2316

55. Huber W, von Heydebreck A, Sultmann H, Poustka A, Vingron M: Variance stabilization applied to microarray data calibration and to the quantification of differential expression. Bioinformatics 2002, 18(Suppl 1): S96-104.

56. Han J, Huang NN, Kim DU, Kehrl JH: RGS1 and RGS13 mRNA silencing in a human $B$ lymphoma line enhances responsiveness to chemoattractants and impairs desensitization. J Leukoc Biol 2006, 79:1357-1368 
57. Nunes-Xavier C, Roma-Mateo C, Rios P, Tarrega C, Cejudo-Marin R, Tabernero L, Pulido R: Dual-specificity MAP kinase phosphatases as targets of cancer treatment. Anticancer Agents Med Chem 2011, 11:109-132.

58. Challa-Malladi M, Lieu YK, Califano O, Holmes AB, Bhagat G, Murty W, Dominguez-Sola D, Pasqualucci L, Dalla-Favera R: Combined Genetic Inactivation of beta2-Microglobulin and CD58 Reveals Frequent Escape from Immune Recognition in Diffuse Large B Cell Lymphoma. Cancer Cell 2011, 20:728-740.

59. Morin RD, Mendez-Lago M, Mungall AJ, Goya R, Mungall KL, Corbett RD, Johnson NA, Severson TM, Chiu R, Field M, et al: Frequent mutation of histone-modifying genes in non-Hodgkin lymphoma. Nature 2011, 476:298-303.

60. Golay J, Introna M: Chemokines and antagonists in non-Hodgkin's lymphoma. Expert Opin Ther Targets 2008, 12:621-635.

61. O'Callaghan K, Lee L, Nguyen N, Hsieh MY, Kaneider NC, Klein AK, Sprague K, Van Etten RA, Kuliopulos A, Covic L: Targeting CXCR4 with cellpenetrating pepducins in lymphoma and lymphocytic leukemia. Blood 2011, 119(7):1717-1725.

62. Hopken UE, Foss HD, Meyer D, Hinz M, Leder K, Stein H, Lipp M: Upregulation of the chemokine receptor CCR7 in classical but not in lymphocyte-predominant Hodgkin disease correlates with distinct dissemination of neoplastic cells in lymphoid organs. Blood 2002, 99:1109-1116.

63. Vockerodt M, Pinkert D, Smola-Hess S, Michels A, Ransohoff RM, Tesch H, Kube D: The Epstein-Barr virus oncoprotein latent membrane protein 1 induces expression of the chemokine IP-10: importance of mRNA halflife regulation. Int J Cancer 2005, 114:598-605.

64. Sander S, Calado DP, Srinivasan L, Kochert K, Zhang B, Rosolowski M, Rodig SJ, Holzmann K, Stilgenbauer S, Siebert R, et al: Synergy between PI3K Signaling and MYC in Burkitt Lymphomagenesis. Cancer Cell 2012, 22:167-179.

65. Schmitz R, Young RM, Ceribelli M, Jhavar S, Xiao W, Zhang M, Wright G, Shaffer AL, Hodson DJ, Buras E, et al: Burkitt lymphoma pathogenesis and therapeutic targets from structural and functional genomics. Nature 2012, 490(7418):116-20.

66. Belardo G, Piva R, Santoro MG: Heat stress triggers apoptosis by impairing NF-kappaB survival signaling in malignant B cells. Leukemia 2010, 24:187-196.

67. Schrader A, Bentink S, Spang R, Lenze D, Hummel M, Kuo M, Arrand JR, Murray PG, Trumper L, Kube D, Vockerodt M: High myc activity is an independent negative prognostic factor for diffuse large $B$ cell lymphomas. Int J Cancer 2011, 131(4):E348-61.

68. Friday BB, Adjei AA: Advances in targeting the Ras/Raf/MEK/Erk mitogenactivated protein kinase cascade with MEK inhibitors for cancer therapy. Clin Cancer Res 2008, 14:342-346.

69. Dai B, Zhao XF, Mazan-Mamczarz K, Hagner P, Corl S, El Bahassi M, Lu S, Stambrook PJ, Shapiro P, Gartenhaus RB: Functional and molecular interactions between ERK and CHK2 in diffuse large B-cell lymphoma. Nat Commun 2011, 2:402.

70. Cheng S, Coffey G, Zhang XH, Shaknovich R, Song Z, Lu P, Pandey A, Melnick AM, Sinha $U$, Wang YL: SYK inhibition and response prediction in diffuse large B-cell lymphoma. Blood 2011, 118:6342-6352.

71. Friedberg JW, Sharman J, Sweetenham J, Johnston PB, Vose JM, Lacasce A, Schaefer-Cutillo J, De Vos S, Sinha R, Leonard JP, et al: Inhibition of Syk with fostamatinib disodium has significant clinical activity in nonHodgkin lymphoma and chronic lymphocytic leukemia. Blood 2010, 115:2578-2585.

72. Witzig TE, Gupta M: Signal transduction inhibitor therapy for lymphoma. Hematology Am Soc Hematol Educ Program 2010, 2010:265-270.

73. Naylor TL, Tang H, Ratsch BA, Enns A, Loo A, Chen L, Lenz P, Waters NJ, Schuler W, Dorken B, et al: Protein kinase $C$ inhibitor sotrastaurin selectively inhibits the growth of CD79 mutant diffuse large B-cell lymphomas. Cancer Res 2011, 71:2643-2653.

74. Chaiwatanatorn K, Stamaratis G, Opeskin K, Firkin F, Nandurkar H: Protein kinase C-beta II expression in diffuse large B-cell lymphoma predicts for inferior outcome of anthracycline-based chemotherapy with and without rituximab. Leuk Lymphoma 2009, 50:1666-1675.

75. Smith SM, van Besien K, Karrison T, Dancey J, McLaughlin P, Younes A, Smith S, Stiff P, Lester E, Modi S, et al: Temsirolimus has activity in non- mantle cell non-Hodgkin's lymphoma subtypes: The University of Chicago phase II consortium. J Clin Oncol 2010, 28:4740-4746.

76. Irizarry RA, Ooi SL, Wu Z, Boeke JD: Use of mixture models in a microarray-based screening procedure for detecting differentially represented yeast mutants. Stat Appl Genet Mol Biol 2003, 2.

77. Hoaglin DC: Understanding Robust and Exploratory Data Analysis. New York, USA: John Wiley \& Sons; 1977.

\section{doi:10.1186/1478-811X-10-43}

Cite this article as: Schrader et al:: Global gene expression changes of in vitro stimulated human transformed germinal centre B cells as surrogate for oncogenic pathway activation in individual aggressive $B$ cell lymphomas. Cell Communication and Signaling 2012 10:43.

\section{Submit your next manuscript to BioMed Central and take full advantage of:}

- Convenient online submission

- Thorough peer review

- No space constraints or color figure charges

- Immediate publication on acceptance

- Inclusion in PubMed, CAS, Scopus and Google Scholar

- Research which is freely available for redistribution 